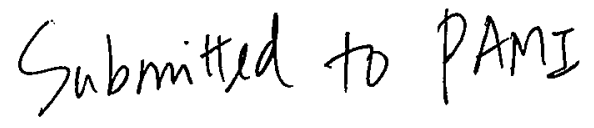

\title{
On Deformable Models for Visual Pattern Recognition
}

\author{
Kwok-Wai Cheung*, Dit-Yan Yeung, and \\ Roland T. Chin* \\ Technical Report HKUST-CS98-08 \\ May 1998 \\ * HKUST \\ Department of Computer Science \\ University of Science \& Technology \\ Clear Water Bay, Hong Kong \\ \{william, dyyeung, roland\}@cs.ust.hk
}

\begin{abstract}
This paper reviews model-based methods for non-rigid shape analysis and recognition. These methods model, match and classify non-rigid shapes, which are generally problematic for conventional algorithms using rigid models. Issues including deformable model representation, optimization criteria formulation, model matching, and classification are examined in detail. The emphasis of this paper is on $2 \mathrm{D}$ deformable models, but some existing $2 \frac{1}{2} \mathrm{D}$ and $3 \mathrm{D}$ deformable models are also briefly reviewed. Potential applications of deformable models and future research directions are discussed.
\end{abstract}




\section{Introduction}

\subsection{Deformable Model-Based Recognition}

Model-based recognition is a process in which an a priori model is searched for in an input image and subsequently its occurrence and location are determined. This approach has been successfully applied to recognize rigid objects, such as machinery parts and printed characters under noisy environment. However, its performance degrades significantly if the shapes to be recognized are non-rigid, such as human faces, cells, gestures and handwritten characters (Figure 1). In order to extend this approach to recognize non-rigid shapes, multiple models could be used for each shape in order to represent different possible deformations. However, such an extension is generally not feasible due to the requirement of a large model set, implying a high computational cost, yet still with no guarantee that all possible deformations be taken care of.

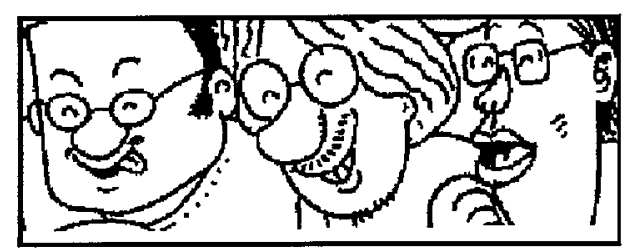

(a) Human faces

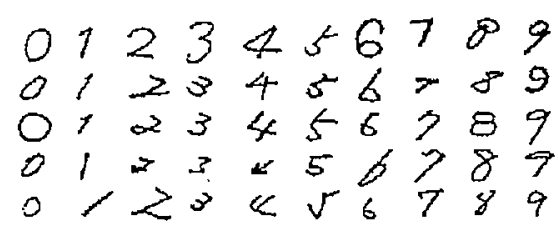

(b) Handwritten characters

Figure 1: Examples of non-rigid shapes

Deformable models (DM) ${ }^{2}$ on the other hand, possess shape-varying ability, which makes them suitable for representing non-rigid patterns. By matching DMs to imagery data, target shapes with possible deformations can be extracted. As a result, multi-class classification is feasible by defining a set of DMs, each containing its own pertinent shape information with an allowable range of deformation specified using a priori information or by training.

They are also often called flexible models. 


\subsection{A Common Formulation}

For the sake of subsequent discussion, a common formulation for DM-based recognition is first introduced here.

\subsubsection{Modeling}

Let $\mathcal{H}_{j}$ denote a $\mathrm{DM}$ based on a particular representation. The model shape is characterized by a parameter vector $\mathbf{w}$, where the parameter values span the parameter space of $\mathcal{H}_{j}$. The model $\mathcal{H}_{j}$ with a particular parameter vector $\mathbf{w}$ is denoted as $\mathcal{H}_{j}(\mathbf{w})$. Model deformation is then specified by varying $\mathbf{w}$ in $\mathcal{H}_{j}(\mathbf{w})$.

\subsubsection{Matching}

Let $\mathbf{D}$ denote the observed imagery data. Given $\mathbf{D}$ and some initial model parameter vector $\mathbf{w}^{0}$, model matching can be viewed as a search process in the parameter space, resulting in a sequence of $\mathrm{DMs} \mathcal{H}_{j}\left(\mathbf{w}^{0}\right), \mathcal{H}_{j}\left(\mathbf{w}^{1}\right), \ldots, \mathcal{H}_{j}\left(\mathbf{w}^{f}\right)$, where $\mathbf{w}^{f}$ denotes the parameter vector of the finally matched model.

Furthermore, the search process is commonly implemented as a multi-criterion optimization problem, although there are studies $[19,38]$ which use heuristic matching processes instead. The following defines the common optimization criteria involved.

\section{- Model deformation criterion:}

This criterion measures the degree of model deformation, i.e., the discrepancy between the current model $\mathcal{H}_{j}(\mathbf{w})$ and the reference (i.e. undeformed) model $\mathcal{H}_{j}(\overline{\mathbf{w}})$, where $\overline{\mathbf{w}}$ is the parameter setting of the reference model. This function is denoted as $E_{\text {def }}\left(\mathbf{w} ; \mathcal{H}_{j}, \overline{\mathbf{w}}\right)$, which represents the "energy" of the current model with respect to the reference model.

- Data mismatch criterion: 
This criterion measures the degree of data mismatch, i.e., the discrepancy between the current model $\mathcal{H}_{j}(\mathbf{w})$ and the given data $\mathbf{D}$. This function is denoted as $E_{m i s}\left(\mathbf{w} ; \mathcal{H}_{j}, \mathbf{D}\right)$, which represents the "energy" of the data with respect to the current model. ${ }^{3}$

\section{- Combined criterion function:}

By combining the model deformation and data mismatch criteria, the "total energy" of the current stage of deformation given the data and the reference model is defined as $E_{\text {tot }}\left(\mathbf{w}, \alpha ; \mathcal{H}_{j}, \mathbf{D}, \overline{\mathbf{w}}\right)=\mathcal{Y}\left(E_{\text {def }}\left(\mathbf{w} ; \mathcal{H}_{j}, \overline{\mathbf{w}}\right), E_{m i s}\left(\mathbf{w} ; \mathcal{H}_{j}, \mathbf{D}\right), \alpha\right)$ where $\alpha$ is a regularization parameter used to regulate the significance of model deformation relative to that of data mismatch. The function $\mathcal{Y}$ denotes a specific combination rule, for example, simple summation or weighted averaging. The matching process thus attempts to minimize the total energy $E_{\text {tot }}$ to obtain the final solution $\mathbf{w}=\mathbf{w}^{f}$ using $\mathcal{H}_{j}$ and $\overline{\mathbf{w}}$ as a priori information about the model.

\subsubsection{Classification}

Let $\mathcal{H}=\left\{\mathcal{H}_{1}, \mathcal{H}_{2}, \ldots \mathcal{H}_{M}\right\}$ denote a set of $M$ different DMs. Classification can be formulated as direct comparison of the model candidates based on the discriminant measure $\mathcal{Z}\left(\mathbf{w}, \alpha ; \mathcal{H}_{j}, \mathbf{D}, \overline{\mathbf{w}}\right)$ associated with each $\mathcal{H}_{j}$. The model with the minimum value of $\mathcal{Z}\left(\mathbf{w}, \alpha ; \mathcal{H}_{j}, \mathbf{D}, \overline{\mathbf{w}}\right)$ is taken as the classified output. If the matching and classification processes share the same criterion, i.e., $\mathcal{Z}=\mathcal{Y}$, then pattern matching and classification may simply be implemented as one single integrated step.

In the literature, the data mismatch criterion is often defined to take also negative values. In that case, the criterion should be interpreted as a relative measure in the sense that its value is only meaningful when compared with another value. 


\subsection{An Example}

To illustrate the application of DMs for pattern matching and shape classification, a twoclass example consisting of a deformable square and a deformable circle is discussed here.

Two models, one for the square and the other for the circle, are matched independently to the given imagery data. Each model $\mathcal{H}_{j}$ is parameterized by an ordered set of $N$ points $\left\{\mathbf{v}_{i}\right\}$, i.e., $\mathbf{w}=\left\{\mathbf{v}_{i}\right\}$. The model deformation criterion is defined as

$$
E_{\text {def }}\left(\mathbf{w} ; \mathcal{H}_{j}, \overline{\mathbf{w}}\right)=\frac{1}{N} \sum_{i=1}^{N}\left\|\mathbf{v}_{i}-\overline{\mathbf{v}}_{i}^{j}\right\|^{2}
$$

where $\overrightarrow{\mathbf{w}}=\left\{\overline{\mathbf{v}}_{i}^{j}\right\}$ is the reference parameter vector of $\mathcal{H}_{j}$ and $\mathbf{w}=\left\{\mathbf{v}_{i}\right\}$ is the current one. Any large shape deviation from the reference shape $\overline{\mathbf{w}}$ will result in a large energy value of $E_{d e f}$. Thus, minimizing $E_{d e f}$ with respect to w will result in a shape that is very close to $\overline{\mathbf{w}}$. For the data mismatch criterion, it is defined as

$$
E_{m i s}\left(\mathbf{w} ; \mathcal{H}_{j}, \mathbf{D}\right)=-\frac{1}{N} \sum_{i=1}^{N}\left\|\mathcal{G}\left(\mathbf{v}_{i} ; \mathbf{D}\right)\right\|^{2}
$$

where $\mathcal{G}\left(\mathbf{v}_{i} ; \mathbf{D}\right)$ is the gradient vector of the input imagery data $\mathbf{D}$ at $\mathbf{v}_{i}$. For a shape $\mathbf{w}=\left\{\mathbf{v}_{i}\right\}$ that falls along an object boundary, $E_{\text {mis }}$ will be minimized. Thus, minimizing $E_{m i s}$ with respect to $\mathrm{w}$ will result in a shape that lies near the boundary, but will be very sensitive to noise. By combining the two criterion functions given in the following

$$
E_{t o t}\left(\mathbf{w}, \alpha ; \mathcal{H}_{j}, \mathbf{D}, \overline{\mathbf{w}}\right)=\frac{1}{N}\left(\alpha \sum_{i=1}^{N}\left\|\mathbf{v}_{i}-\overline{\mathbf{v}}_{i}^{j}\right\|^{2}-\sum_{i=1}^{N}\left\|\mathcal{G}\left(\mathbf{v}_{i} ; \mathbf{D}\right)\right\|^{2}\right)
$$

and minimizing $E_{t o t}$ with respect to $\mathbf{w}$, the resulting $\mathbf{w}$ will be a compromise between conforming to the reference model $\overline{\mathbf{w}}$ and conforming to the given data $\mathbf{D}$. In this illustrative example, a greedy algorithm for the minimization with guaranteed convergence is used for simplicity, i.e., $\mathbf{v}_{i}$ is updated one at a time and the iteration repeats until all the points have converged. Details of the algorithm are shown below. 
Total $:=\infty$
$\mathbf{w}:=\mathbf{w}^{0}$

do

$$
\begin{aligned}
& \text { Last_Total }:=\text { Total } \\
& \text { Total }:=0
\end{aligned}
$$

for all the points $\mathbf{v}_{i}$,

for all the neighbors $N_{j}\left(\mathbf{v}_{i}\right)$ of $\mathbf{v}_{i}$,

$$
\begin{aligned}
& E_{\text {def }}\left(N_{j}\left(\mathbf{v}_{i}\right)\right):=\left\|N_{j}\left(\mathbf{v}_{i}\right)-\overline{\mathbf{v}}_{i}\right\|^{2} \\
& E_{\text {mis }}\left(N_{j}\left(\mathbf{v}_{i}\right)\right):=-\left\|\mathcal{G}\left(N_{j}\left(\mathbf{v}_{i} ; \mathbf{D}\right)\right)\right\|^{2} \\
& E_{\text {tot }}\left(N_{j}\left(\mathbf{v}_{i}\right)\right):=\alpha E_{\text {def }}\left(N_{j}\left(\mathbf{v}_{j}\right)\right)+E_{m i s}\left(N_{j}\left(\mathbf{v}_{j}\right)\right)
\end{aligned}
$$

endfor.

Total $:=$ Total $+\min _{j}\left\{E_{\text {tot }}\left(N_{j}\left(\mathbf{v}_{i}\right)\right)\right\}$

update $\mathbf{v}_{i}$ by $N_{j}\left(\mathbf{v}_{i}\right)$ with minimum $E_{\text {tot }}\left(N_{j}\left(\mathbf{v}_{i}\right)\right)$

endfor.

until Last_Total - Total converges to zero.
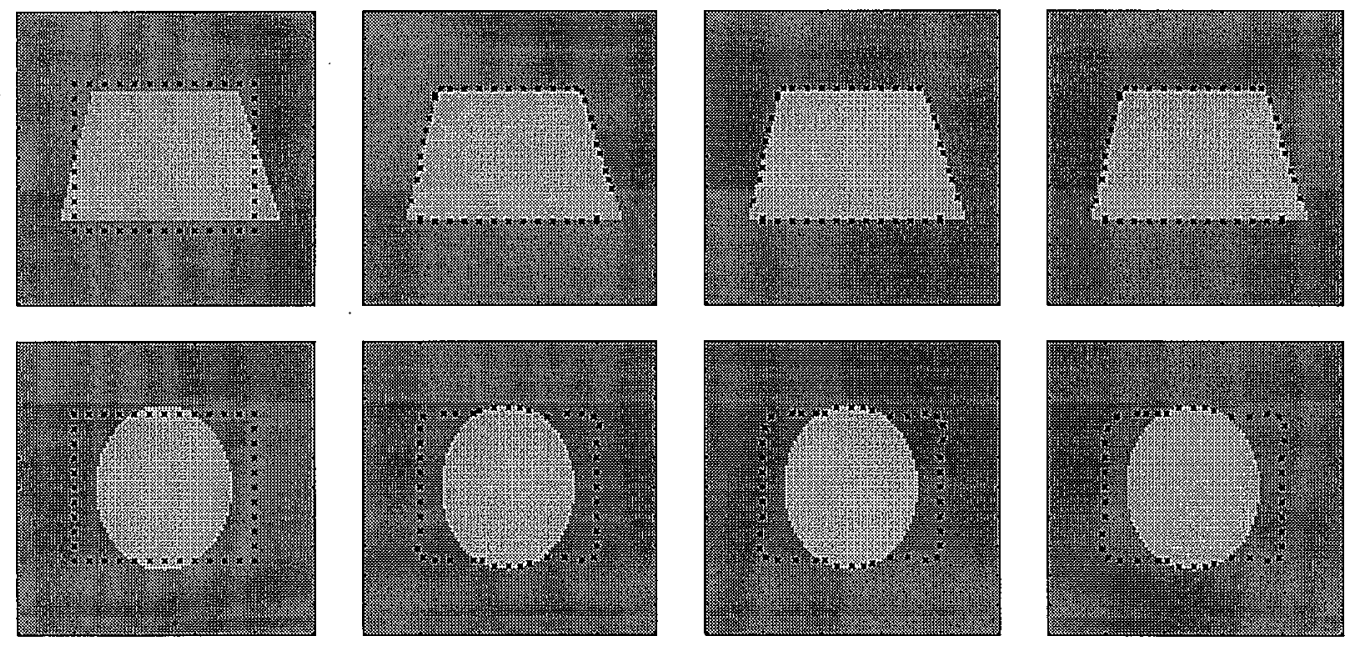

Figure 2: The results of matching a deformable square model to a trapezoidal image (upper row) and an elliptical image (lower row). The initial model with $\mathbf{w}^{0}=\overline{\mathbf{w}}$ is shown as black dots in the leftmost column. The square deforms from left to right to match with the object boundary as a compromise between preserving $\overline{\mathbf{w}}$ and matching to the underlying data.

Figures 2 and 3 illustrate the matching results of the deformable square and the deformable circle, respectively. As illustrated in the figures, the model deforms and tries to 

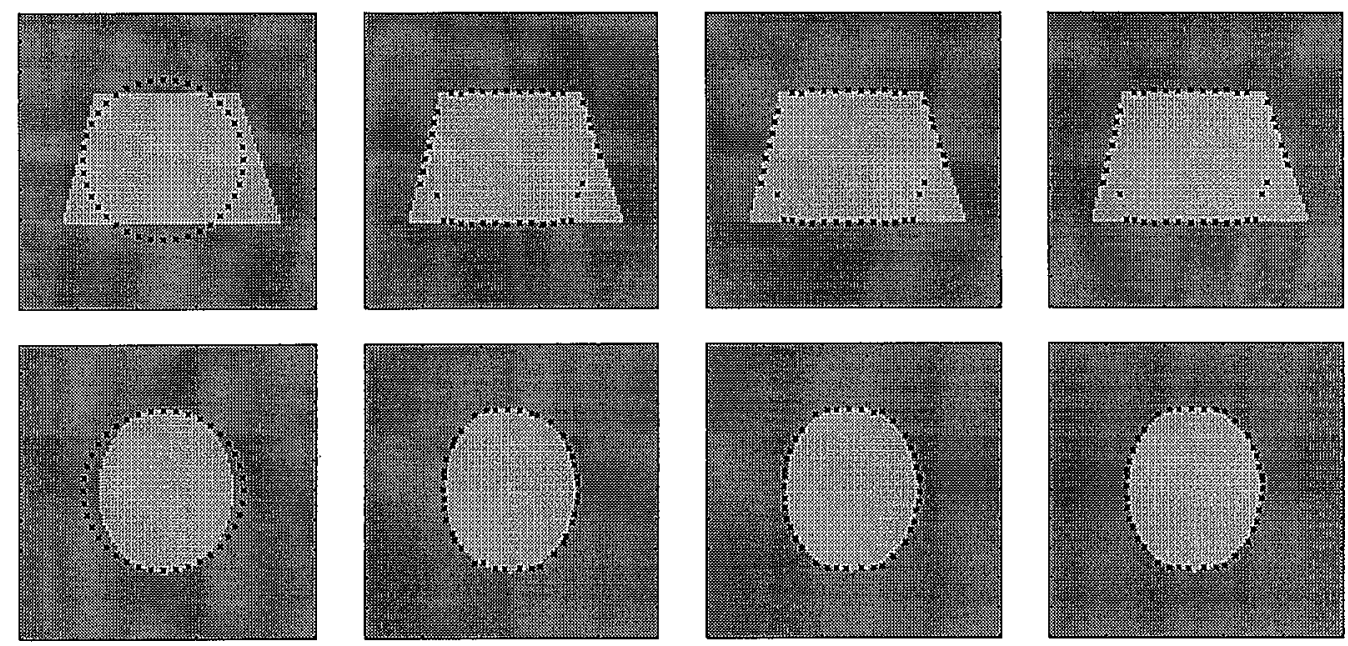

Figure 3: The results of matching a deformable circle model to a trapezoidal image (upper row) and an elliptical image (lower row).

match with the underlying data until it converges. Note that the trapezoidal (elliptical) shape can only be successfully extracted by the corresponding deformable square (circle) model. The values of the three energy criterion functions before the minimization and after the convergence are tabulated in Table 1.

\begin{tabular}{|c|c||c|c||c|c|}
\hline \multicolumn{2}{|c||}{} & \multicolumn{2}{c||}{ Square Model } & \multicolumn{2}{c|}{ Circle Model } \\
\cline { 2 - 6 } \multicolumn{2}{|c|}{} & Before & After & Before & After \\
\hline \multirow{4}{*}{ Trapezoidal Image } & $E_{\text {def }}$ & 0 & 9.5 & 0 & 10.92 \\
\cline { 2 - 6 } & $E_{\text {mis }}$ & 0 & -0.81 & 0 & -0.75 \\
\cline { 2 - 6 } & $E_{\text {tot }}$ & 0 & -0.80 & 0 & -0.74 \\
\hline \multirow{4}{*}{ Elliptical Image } & $E_{\text {def }}$ & 0 & 9.77 & 0 & 11.66 \\
\cline { 2 - 6 } & $E_{\text {mis }}$ & 0 & -0.28 & 0 & -0.72 \\
\cline { 2 - 6 } & $E_{\text {tot }}$ & 0 & -0.23 & 0 & -0.66 \\
\hline
\end{tabular}

Table 1: Values of the three energy criterion functions before the minimization and after the convergence. The value of $\alpha$ is fixed to be a constant in this example.

Using $E_{\text {tot }}$ as discriminant measure, it is noted that the deformed square model has 
smaller discrepancy than the deformed circle model for the trapezoidal image, while the deformed circle model has smaller discrepancy than the deformed square model for the elliptical image. Therefore, the trapezodial and the elliptical images are classified as "square" and "circle", respectively.

\subsection{Development Milestones}

The development of DM-based recognition has a history of about two decades. To the best of our knowledge, the earliest work using the idea of shape-varying models for image analysis is the study of rubber masks by Widrow [140] in 1973 to analyze and recognize non-

rigid objects, like chromosomes and noisy waveforms. With a similar idea, Fischler et al. [54] proposed the spring model which is composed of different shape primitives connected by springs. By stretching and shrinking the springs, the spatial relationship between the shape primitives, and thus the overall shape, can be varied. Elastic matching proposed by Burr $[19,18]$ in 1981 defines some displacement vectors based on heuristics which direct the deformation of a skeleton model to match with the input data.

The study of DMs blossomed recently due to the work of active contour models (or snakes) proposed by Kass et al. [77] in 1988, where DM matching is formulated as optimization of a combined criterion with an internal energy (i.e. model deformation) term $E_{\text {int }}$ and an external energy (i.e. data mismatch) term $E_{\text {ext }}$. The optimization process can be equivalently represented by the dynamics of a physical process, where $\partial E_{\text {int }} / \partial \mathbf{w}$ acts as internal force and $\partial E_{\text {ext }} / \partial \mathbf{w}$ as external force. The model will then deform and settle at an equilibrium point of the dynamical system. Thus, such active models are also referred to as physically-based models. Compared with purely geometrically-based approaches, the novelties of physically-based models include using an internal energy term that governs model deformation and introducing an optimization framework with a corresponding physical interpretation. Through a graphical user interface, a "snake", when initialized in location 
properly, was demonstrated to be able to jigger on an image and finally latch on some object boundary. The robustness and the dynamic nature of the snake model make it attractive for noisy and subjective boundary detection as well as motion tracking. ${ }^{4}$ Based on the work of active contour models, many extensions have been proposed to achieve better matching and more efficient implementation. Besides, different $2 \frac{1}{2} \mathrm{D}$ and $3 \mathrm{D}$ DMs have also been proposed for image or volumetric data analysis $[132,139,131]$.

Most of the existing works on DMs are purely based on an optimization framework, which in fact can have a probabilistic interpretation. Introducing probabilistic interpretation to computer vision tasks, e.g., boundary detection, can be dated back to Cooper's early work [37] in 1979, where a maximum likelihood method was proposed for detecting blob boundaries in noisy images. In 1992, Staib et al. [127] formulated a DM matching problem as maximum a posteriori (MAP) estimation using Bayesian techniques. In the neural network community, Durbin and Willshaw [52] in 1987 proposed the elastic net model to solve the Traveling Salesman Problem. The idea is very much like using a deformable contour to match to a set of points. The probabilistic interpretation of the elastic net has also been proposed [51]. Later, Yuille [150] generalized this interpretation to include different artificial neural network models and DMs.

The importance of the probabilistic interpretation is more than just a nice mathematical framework. It suggests that lots of techniques for parameter estimation and hypothesis testing can be borrowed from the statistical literature to achieve matching and classification in a more disciplined manner. Revow et al. [118] proposed to perform DM matching using the expectation-maximization (EM) algorithm [47], which is originated from statistics for maximum likelihood estimation (MLE) problems with missing data. Besides, they also combined the probabilistic interpretation with MacKay's evidence framework [95] and

In computer graphics, such physically-based models are also very useful for producing realistic animation of the interaction of non-rigid natural objects. Interested readers are referred to [130] for further details. 
formulated a DM-based classification problem, previously formulated in a heuristic manner, formally as a model selection problem.

\begin{tabular}{|c|c|c|c|}
\hline References & Year & Proposed Model & Remarks \\
\hline "Widrow [140] & 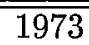 & Rubber mask & \multirow{3}{*}{ Early work } \\
\hline Fischler et al. [54] & 1973 & Spring model & \\
\hline Burr $[19,18]$ & 1981 & Elastic matching & \\
\hline "Kass et al. [77] & 1987 & $\begin{array}{l}\text { Active contour model } \\
\text { (Snake) }\end{array}$ & $\begin{array}{l}\text { Energy minimization problem } \\
\text { (optimization, regularization) }\end{array}$ \\
\hline Durbin et al. [52] & 1987 & Elastic net & $\begin{array}{l}\text { Energy minimization problem } \\
\text { (optimization, regularization) }\end{array}$ \\
\hline Durbin et al. [51] & 1989 & Elastic net & $\begin{array}{l}\text { Probabilistic interpretation } \\
\text { of elastic net }\end{array}$ \\
\hline Yuille [150] & 1990 & $\begin{array}{l}\text { Generalized deformable } \\
\text { model }\end{array}$ & $\begin{array}{l}\text { Generalization and unification } \\
\text { of some existing models }\end{array}$ \\
\hline Revow et al. [118] & 1996 & $\begin{array}{l}\text { Elastic spline } \\
\text { model }\end{array}$ & $\begin{array}{l}\text { Combining the probabilistic } \\
\text { interpretation of elastic net with } \\
\text { MacKay's Bayesian framework } \\
\text { to formulate the classification problem } \\
\text { as a model selection problem }\end{array}$ \\
\hline
\end{tabular}

Table 2: Major milestones

In the literature, there also exist other related matching algorithms like relaxation labeling [32] and self-organizing map [92]. Since their formulations are quite different from the common framework described in Section 1.2, they will not be further described in this paper.

\subsection{Paper Organization}

The emphasis of this survey paper is on using 2D DMs for visual pattern recognition. Representative works proposed in the literature are categorized and compared based on their model representation (Section 2), criterion function formulation (Section 3), regularization (Section 4), matching (Section 5) and classification (Section 6) techniques. Also, some existing $2 \frac{1}{2} \mathrm{D}$ and $3 \mathrm{D}$ DMs will also be briefly discussed in Section 7. Potential applications of DMs proposed in the literature are summarized in Section 8. Finally, some future research directions are discussed in Section 9. The paper organization is summarized in Table 3. 
1 Introduction

1.1 Deformable Model-Based Recognition

1.2 A Common Formulation

1.3 An Example

1.4 Development Milestones

1.5 Paper Organization

2 Model Representation

\subsection{Parameterization}

2.2 Constraint Incorporation

2.3 Geometric Invariance

3 Criterion Function Formulation

3.1 Model Deformation Criterion

3.2 Data Mismatch Criterion

3.3 User-Controlled Criterion

4 Regularization

5 Matching

5.1 Initialization

5.2 Optimization

5.3 Probabilistic Interpretation

6 Classification

6.1 Representation Uniqueness

6.2 Discriminant Measures for Classification

$72 \frac{1}{2} \mathrm{D}$ and 3D Deformable Models

8 Applications

9 Future Research Directions

10 Conclusion

References

Table 3: Table of Contents 


\section{Model Representation}

The first most crucial step that determines the success of DM-based algorithms is the choice of a good model representation. The criteria for measuring how good a representation is [116] include:

Representational power The representation should be general enough to model all the objects of interest in some specific application domains, and yet is not so general that over-generalization to some undesirable objects would be resulted.

Uniqueness Representation uniqueness refers to the property that no two instances of a representation can model a shape equally well. This property is important for simplifying shape comparison.

Stability Small changes in the representation should result in only small changes in the overall shape.

Efficiency The computational complexity for both computing the representation given the input data and comparing different representations should not be too high.

These considerations will be taken into account in the following discussions for comparing different representations. To categorize different approaches to model representation, a "non-parametric to parametric" spectrum will be used.

\subsection{Parameterization}

\subsubsection{A Spectrum Characterizing the Degree of Parameterization}

In traditional statistical pattern recognition [61] (and in fact for statistics in general), parametric and non-parametric approaches form two distinct branches. The parametric approach typically assumes that the underlying probability density function of the input data can be compactly represented in some analytical form which is parameterized by a small set 
of parameters, such as mean and variance. On the other hand, the non-parametric approach does not assume any analytical form but the density function is represented explicitly using the data. In practice, the distinction is fuzzy. There exist methods where the number of parameters is so large and the parameters are not so interpretable that these methods do not have the common notion of being parametric. To elaborate this point, let us consider an example of representing a circle in two dimensions. A circle can be represented either by specifying its centre and radius, or by using an ordered set of points to be interpolated. Both representations can be said to be parametric. However, the first representation requires just three parameters to represent a circle perfectly while the second one requires a larger number of points as parameters for precise representation of a circle. In the extreme case when the number of points equals the number of pixels forming the circle, the representation becomes purely non-parametric. This suggests that the distinction between non-parametric and parametric approaches is not well defined, but a continuum exists between the two extreme cases. However, for the sake of further discussion, models with "many" parameters are referred to as non-parametric here while those with compact analytical form characterized by a small set of parameters are referred to as parametric. Also, models with mixed representation types are referred to as semi-parametric. See Figure 4 for a pictorial illustration.

\subsubsection{Non-parametric Models}

The simplest way to represent a shape, say the silhouette of an object, is through piecewise linear approximation by linearly interpolating an ordered set of points. This is categorized

as a non-parametric technique in this paper. Kass et al.'s active contour model [77] is a typical example, where a set of interpolated points forms the representation of a contour. Such a representation has also been adopted by many other researchers in the field [58, $36,91,143,2,90,82,84]$. With a sufficient number of model points, an active contour 


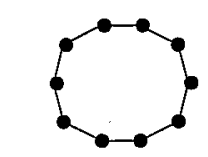

Piecewise linear curve

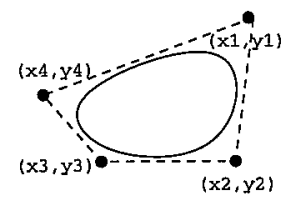

B-spline curve

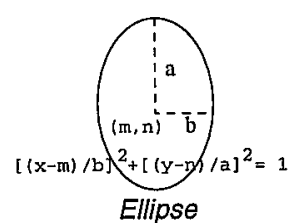

Parametric model

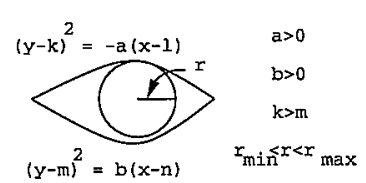

Eye template

Parametric model with explicit constraints

Non-parametric models

Semi-parametric model

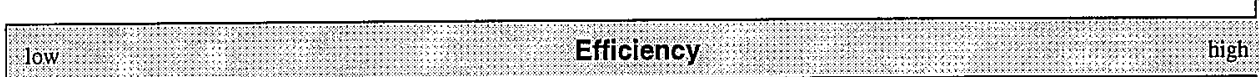

.

ㄱ.
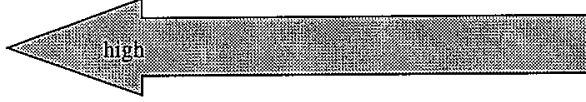

Representational power.

Figure 4: Examples of models with different degrees of parameterization

model can represent any arbitrary shape accurately, implying high representational power of the model. However, such a simple representation is not cost-effective for smooth shapes, like circles, where a large number of model points are required to achieve negligible sawtooth effect. Also, as it is basically a free-form model, i.e., not specific to any particular shape, its uniqueness property is not well considered, hindering it from direct application to recognition. By and large, it is still a DM commonly used for boundary detection and motion tracking. Other than using contours, Lades et al. [81] used a 2D regular attributed ${ }^{5}$ grid to represent human faces and proposed labeled graph matching. The grid representation was later enhanced to a fiducial graph, a specially designed graph triangulation with grid points (graph node) located at anatomically identifiable points of the face for a more accurate representation. Due to the use of attributes, it is a feature-based representation. See Section 5.1 for more discussions.

The attributes on the grid points are the responses of multi-scale multi-orientation Gabor wavelets applied to a reference face. In this sense, it is a feature-based model. 


\subsubsection{Parametric Models}

To alleviate the efficiency limitation of non-parametric model representations, models with some analytical forms can be used. A carefully designed parametric model, characterized by just a small number of shape parameters, may also have good representational power. An implicit polynomial [129] is a good example which uses the zero sets of some algebraic functions (i.e., the set of solutions for the functions to take zero value) to represent arbitrary shapes. Furthermore, a parametric model based on elliptic Fourier decomposition of contours has been proposed [127], where other than computational advantages, Fourier decomposition provides an orthogonal basis which makes the representation unique. A unique representation can simplify the problem of formulating a good model deformation criterion function $E_{d e f}$ (see Section 3.1) for model shape comparison. More discussions on the importance of representation uniqueness for shape comparison and the usage of orthogonal parameterization can be found in $[116,114]$.

\subsubsection{Semi-parametric Models}

Although using parametric models seems to be a better choice, they are mostly restricted to the representation of relatively smooth and continuous shapes. To enjoy the advantages of both non-parametric and parametric approaches, a hybrid approach, which will be referred to as the semi-parameteric approach, can be adopted. Good examples are Bézier [30] or Bspline curves $[69,71,28,118]$. Using a small set of control points (non-parametric portion), a smooth and continuous contour can easily be represented by a cubic B-spline curve (with the B-spline blending functions being the parametric portion) and is guaranteed to be $C^{0}, C^{1}$ and $C^{2}$ continuous [56]. Besides, another semi-parametric representation has been proposed for modeling shapes using the finite element method $[123,124]$. The representation consists of a wireframe, which is a mesh of interconnected nodes with fixed topology (non-parametric 
portion) and its physical properties are defined by a mass matrix, a material stiffness matrix, a damping matrix and an interpolation matrix (parametric portion). The wireframe can allow the model to have good representational power (due to its non-parametric nature) while the interpolation matrix, which interpolates the properties of any arbitrary point based on its neighboring nodes, acts as a local parametric model to keep the number of wireframe nodes small (due to the parametric nature) and hence improves the model parsimony.

\subsection{Constraint Incorporation}

\subsubsection{Constraints on Model Parameters}

Most of the model representations mentioned above possess free-form structures, where the model parameters can vary freely to model arbitrary shapes. However, in many applications, only some restricted classes of shapes are of interest and hence free-form structures may be undesirable, partly due to the corresponding large search space. For example, to locate the eyes in a human face image using a deformable eye model, one would expect that the deformed model should remain as an eye-like shape but not some other shape. In that case, the DM should have constraints, which can be incorporated either implicitly or explicitly.

The deformable template used by Yuille et al. [151, 152] consists of parametric curve components, where constraints are incorporated implicitly through parametric forms. For example, an eye template used in [151] consists of two parabolic curves modeling the upper and lower eye lips and one circle modeling the iris. Thus, the DM is parameterized by the coefficients of the upper and lower parabolas together with the centre and radius of the circle. For explicit constraints, the upper lid is restricted to be always convex upwards and the lower lid to be always convex downwards. This is done by confining the parameter space. For other examples of incorporating implicit constraints, Bascle et al. [5] defined some parametric features such as corners and junctions to achieve robust feature 
extraction. Cootes et al.'s point distribution model (PDM) [38] uses a set of contour components, each defined by an ordered set of points (also called "landmarks") to represent human faces. The model is represented by a linear combination of basis vectors, obtained by eigen-decomposition of the landmarks' covariance matrix (Figure 5). The notion of 'distribution' is due to the assumption that the set of ordered points follows a multivariate Gaussian distribution. Constraints can be introduced by trimming off the basis vectors with less significant eigenvalues, which correspond to relatively rare types of deformation. Further enhancement in PDM was suggested by assuming that the intensity gradient profiles perpendicular to the contours follow another multivariate Gaussian distribution [40]. ${ }^{6}$ Similar decomposition was also adopted by Sclaroff et al. [124], but on the stiffness matrix instead of the covariance matrix. Decomposition of the stiffness matrix results in a set of vibration modes with different "frequencies" of vibration. The high frequency components corresponding to highly distorted shapes are assumed to be relatively rare and hence are trimmed off.

For the examples of explicit constraint incorporation, Dubuisson et al. [50] used a set of hand-drafted constraints to explicitly restrict the deformation of a deformable polygon for representing cars of different types. Besides, Olstad et al. [111] used syntactical approaches to incorporate constraints, where a priori information about object shape can be encoded into an active contour model as a set of grammar rules. Also, Fua et al. [57] confined the model parameter search in the orthogonal subspaces of some given hard constraint surfaces.

Hard-wiring constraints into models manually, in most cases, cannot be done very accurately. Taking PDM as an example, it is difficult to determine accurately the mean vector and covariance matrix of the landmarks for each class. Training is usually required. The

Using a multivariate Gaussian is basically to assume the distribution of the parameters to be unimodal. In general, for better modeling, some multimodal distributions, e.g., mixture of multivariate Gaussians, can be used. 


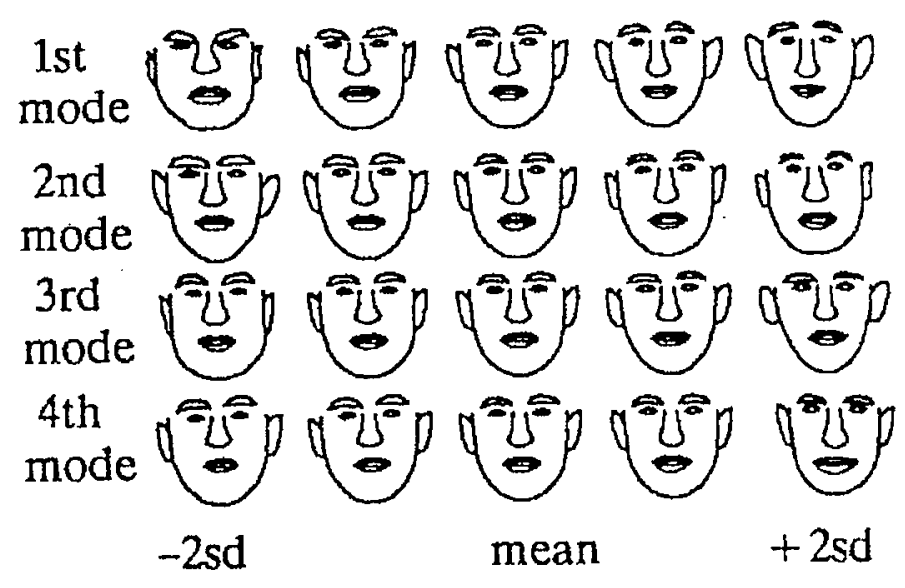

Figure 5: Different principal components obtained by Cootes et al. [4] for a deformable face model

major disadvantage of training is the requirement of large quantity of clean training data. Creating a large training set very often demands tremendous human effort, as one may need to manually locate well-matched models for all the training cases. The situation is even worse for cases when only limited training data are available. To remedy this limitation, one may start with a coarse initial model. Subsequent training that follows will lead to model refinement, which usually requires much less data than the case if the model is trained from scratch. Such techniques have been studied by Cootes et al. [39] for the estimation of covariance matrix and found to be quite effective.

\subsubsection{Constraints via Deformation Transformation}

To deform the shape of a model, instead of varying its model parameters independently, some global transformation may also be applied to the whole model. Affine transformation is one example (see Section 2:3 for more details). Since it is mostly used to approximate shape change in viewpoint-independent object recognition, such transformation in general should be permitted, i.e., it should not be penalized in the model deformation criterion (see Section 3.1). More details about achieving this geometric invariance property can be found 


\section{in Section 2.3.}

As global affine transformation is not a good choice for representing arbitrarily deformed shapes, Wakahara [138] proposed a local affine transform (LAT) model. The idea is to have neighboring points of a model to form groups, each sharing a single local affine transform. The new parameterization constrains the model by assuming that neighboring model points can only undergo affine transformation.

Other than linear or piecewise linear transformation, one may even relax the linearity constraint to come up with a non-linear deformation transformation. Global shape models, first proposed by Grenander et al. [63] for analyzing biological shapes, use a global parametric displacement field which is represented by a set of orthogonal basis functions. Each basis function corresponds to one kind of restricted global deformation. Using the displacment field, the deformation of an arbitrary pixelwise template (called anatomical textbook in [100]) can easily be defined as a set of coefficients of the basis functions. Similar ideas have been adopted in $[31,73]$.

\subsection{Geometric Invariance}

The geometric invariance property, though largely ignored in boundary detection, is vital to viewpoint-independent object recognition. If an object is moved along a plane perpendicular to the optical axis of the camera or along that axis, its shape will undergo similarity transformation, which includes rotation, shifting and scaling. If the perpendicular assumption is relaxed and thus the plane is allowed to be tilted, it will result in the projective effect, which can be well approximated by an affine transform that may also include shearing [4] (Figure 6$).{ }^{7}$ By factoring out the underlying transformation, a representation becomes viewpoint-independent, or called geometrically invariant.

It should be noted that, for some applications, it would be better to have restrictions on the affine transform. 


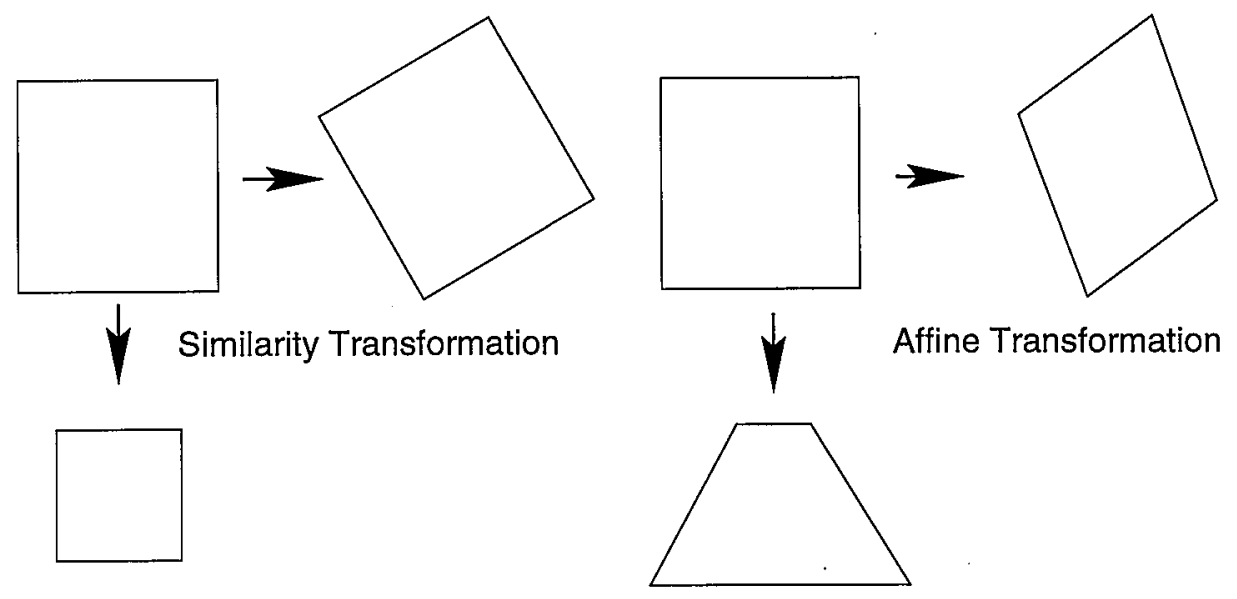

Figure 6: Examples of similarity and affine transformations. A similarity transformation is composed of scaling, rotation and translation while an affine transform also includes shearing.

A geometrically invariant representation can be achieved either implicitly by choosing a geometrically invariant parameterization or explicitly by directly estimating the transformation parameters. Implicit methods have the advantage of estimating a relatively smaller set of parameters and thus are more efficient. Explicit methods, however, can provide pose information of objects immediately after matching, which may be required for further processing. Examples of implicit methods include Lai's generalized active contour models [84], where affine-invariant shape matrices are used to represent different shapes. Besides, Subrahmonia's implicit polynomial representation [129] uses algebraic invariants, which are functions of the implicit polynomial coefficients and are invariant to affine transformation.

Direct estimation of the affine transformation parameters is rather easy to understand. Many examples already exist, e.g. [151, 152, 71, 69]. 


\section{Criterion Function Formulation}

Based on a chosen model representation, the associated model deformation criterion and data mismatch criterion can then be defined. The criterion functions can be interpreted as soft constraints (penalties) restricting model deformation. Many DMs were originally derived using criterion functions with continuous integration. Since discretization is an inevitable step for digital implementations, in the following two sections, only discrete versions of the criterion functions are described, i.e., with all the integrations replaced by summations.

\subsection{Model Deformation Criterion}

A model deformation criterion measures the degree of model deformation. It is also called internal energy in [77], deformation energy in [69], strain energy in [124], etc. Alternatively, it can be interpreted as a penalty for deforming a model. Spatial derivatives of the criterion function can be interpreted as some internal forces exerted on the model to restore it back to its reference (i.e. undeformed) shape. For the example of a contour model, the deformation criterion can be defined as the inter-point distance or the curvature of the contour. Mathematically, they can be formulated as follows.

Let $\mathbf{w}^{t}$ denote the transpose of $\mathbf{w}$ and $\left\{\mathbf{v}_{i}\right\}$ denote a set of coordinates defining a model parameterized by $\mathbf{w}$, i.e., $\mathbf{w}^{t}=\left(\mathbf{v}_{0}^{t}, \mathbf{v}_{1}^{t}, \mathbf{v}_{2}^{t}, \ldots\right)$.

\section{Inter-point distance}

$$
E_{d e f_{1}}\left(\mathbf{w} ; \mathcal{H}_{j}, \overline{\mathbf{w}}\right)=\sum_{i=1}^{k}\left\|\mathbf{v}_{i}-\mathbf{v}_{i+1}\right\|
$$

\section{Curvature}

$$
E_{d e f_{2}}\left(\mathbf{w} ; \mathcal{H}_{j}, \overline{\mathbf{w}}\right)=\sum_{i=1}^{k}\left\|\mathbf{v}_{i-1}-2 \mathbf{v}_{i}+\mathbf{v}_{i+1}\right\|
$$


where $k$ is the dimension of $\mathbf{w}$ and $\|\cdot\|$ denotes a vector norm. In particular, if the $L_{2}$ norm is used, $E_{d e f_{1}}\left(\mathbf{w} ; \mathcal{H}_{j}, \overline{\mathbf{w}}\right)$ and $E_{\text {def }}\left(\mathbf{w} ; \mathcal{H}_{j}, \overline{\mathbf{w}}\right)$ can be expressed as $\mathbf{w}^{t} \mathbf{A} \mathbf{w}$ and $\mathbf{w}^{t} \mathbf{B w}$, respectively, where $\mathbf{A}$ is a tri-diagonal matrix and $\mathbf{B}$ is a penta-diagonal matrix. The spatial derivatives of $E_{d e f_{1}}$ and $E_{d e f_{2}}$ can be interpreted as some internal forces exerted on the model such that the contour points are pulled together and the contour is prevented from bending too much.

The criteria in EQ. (4) and (5) prefer a shorter curve to a longer one with the same curvature. To achieve scale invariance, normalization can be used, e.g., a new criterion can be defined as the ratio of $E_{d e f_{2}}$ to $E_{d e f_{1}}[82]$.

More generally, model deformation can be defined as some distance between the current model parameter vector $\mathbf{w}$ and its reference value $\overrightarrow{\mathbf{w}}$ in a parameter space based on the chosen representation. By assuming a quadratic form, it can be defined as

$$
E_{d e f_{3}}\left(\mathbf{w} ; \mathcal{H}_{j}, \overline{\mathbf{w}}\right)=(\mathbf{w}-\overline{\mathbf{w}})^{t} \mathbf{\Sigma}^{-1}(\mathbf{w}-\overline{\mathbf{w}})
$$

where $\boldsymbol{\Sigma}$ could be a diagonal matrix (resulting in a weighted Euclidean distance in the parameter space), or the covariance matrix of $\mathbf{w}$ learned from a set of training data (resulting in the Mahalanobis distance [141]), or a manually defined stiffness matrix [124, 38]. For physical interpretation, $\boldsymbol{\Sigma}$ being a diagonal matrix basically assumes that the contour points can be moved independently, while $\boldsymbol{\Sigma}$ being the covariance matrix or some other nondiagonal matrix introduces interaction among the contour points.

\subsection{Data Mismatch Criterion}

The data mismatch criterion measures the data discrepancy given the current model. The spatial derivatives of this criterion can be interpreted as some external or image forces exerted on the model to deform it to match some regions of interest of the data. By introducing different types of preprocessing on the input, various data mismatch criteria 
have been proposed and can be categorized into image-based, edge-based, region-based and motion-based formulations. The criteria can be used cooperatively to facilitate good matching $[156,50,24]$.

\subsubsection{Image-Based}

To match a model to pixels with high intensity, the data mismatch criterion [77] can be formulated as

$$
E_{m i s_{1}}\left(\mathbf{w} ; \mathcal{H}_{j}, \mathbf{D}\right)=-\sum_{i=1}^{N_{v}} \mathcal{I}\left(\mathbf{v}_{i}(\mathbf{w}) ; \mathbf{D}\right)
$$

where $\mathcal{I}\left(\mathbf{v}_{i} ; \mathbf{D}\right)$ denotes the intensity of the image $\mathbf{D}$ at $\mathbf{v}_{i}$ and $N_{v}$ denotes the number of elements in $\left\{\mathbf{v}_{i}\right\} .^{8} \mathrm{~A}$ more complicated criterion, with a probabilistic interpretation (see Section 5.3), was proposed in [141] for matching to black pixels in a binary image. It is defined as

$$
E_{m i s_{2}}\left(\mathbf{w} ; \mathcal{H}_{j}, \mathbf{D}\right)=-\sum_{l=1}^{N} \log \frac{1}{N_{v}} \sum_{i=1}^{N_{v}} \frac{\beta}{2 \pi} \exp \frac{-\beta\left\|\mathbf{v}_{i}(\mathbf{w})-\mathbf{y}_{l}\right\|^{2}}{2}
$$

where $\mathrm{y}_{l}$ is the location of an individual black pixel, $\beta$ is a signal-strength parameter and $N$ is the number of black pixels.

\subsubsection{Edge-Based}

Image edges have long been used as salient features for image analysis as they are less sensitive to different lighting conditions. To match a model to image edges where the gradient magnitude is usually large [77], the data mismatch criterion can be formulated as

$$
E_{m i s_{3}}\left(\mathbf{w} ; \mathcal{H}_{j}, \mathbf{D}\right)=-\sum_{i}\left\|\mathcal{G}\left(\mathbf{v}_{i}(\mathbf{w}) ; \mathbf{D}\right)\right\|
$$

where $\mathcal{G}\left(\mathbf{v}_{i} ; \mathbf{D}\right)$ denotes the gradient vector of the image $\mathbf{D}$ at $\mathbf{v}_{i}$. To further utilize the gradient directional information [112], we can define the following criterion

$$
E_{m i s_{4}}\left(\mathbf{w} ; \mathcal{H}_{j}, \mathbf{D}\right)=-\sum_{i}\left|\left(\cos \phi \cos \psi \mathcal{G}_{x}, \sin \phi \sin \psi \mathcal{G}_{y}\right)\right|
$$

For matching to dark pixels, simply remove the negative sign in EQ.(7). 
where $\mathcal{G}_{x}$ and $\mathcal{G}_{y}$ are the $x$ and $y$ components of $\mathcal{G}\left(\mathbf{v}_{i} ; \mathbf{D}\right)$ respectively, $\phi$ is the normal angle of the contour at $\mathbf{v}_{i}$ and $\psi$ is the gradient angle there. Such a formulation tries to avoid matching to some undesirble edges caused by irrelevant objects in the neighborhood. Similar ideas have also been adopted in [146, 83].

Moreover, in order for the model to favor boundaries with a constant gradient [58], the criterion can be formulated as

$$
E_{m i s_{5}}\left(\mathbf{w} ; \mathcal{H}_{j}, \mathbf{D}\right)=-\frac{1}{N_{v}} \sum_{i=1}^{N_{v}}\left|\left\|\mathcal{G}\left(\mathbf{v}_{i}(\mathbf{w}) ; \mathbf{D}\right)\right\|-\overline{\mathcal{G}}\right|
$$

where $\overline{\mathcal{G}}=\frac{1}{L} \sum_{i=1}^{N_{v}}\left\|\mathcal{G}\left(\mathbf{v}_{i}(\mathbf{w}) ; \mathbf{D}\right)\right\|$ is the average gradient magnitude.

\subsubsection{Region-Based}

Other than using edge information, statistical properties of the region bounded by the model can also be used to give better matching [48]. The simplest one adopted in $[120,23]$ has the formulation as

$$
E_{m i s_{6}}\left(\mathbf{w} ; \mathcal{H}_{j}, \mathbf{D}\right)=-\sum_{i=1}^{N_{v}} \mathcal{F}\left(\mathbf{v}_{i}(\mathbf{w}) ; \mathbf{D}\right)
$$

where $\mathcal{F}$ is an indicator function for foreground pixels and can be computed using some standard region-based segmentation algorithms. This criterion tends to direct the model to enclose regions with homogeneous grey level. To model a noisy homogeneous region, a stochastic process can be used with the corresponding criterion formulated as

$$
E_{m i s 7}\left(\mathbf{w} ; \mathcal{H}_{j}, \mathbf{D}\right)=-\sum_{\mathbf{s} \in \mathbf{R}}\left[\left(\frac{\mathcal{I}(\mathbf{s} ; \mathbf{D})-\mu}{\sigma}\right)^{2}-f_{s}\right]
$$

where $\mathbf{R}$ denotes the set of pixels enclosed by the model contour, $\mathcal{I}(\mathbf{s} ; \mathbf{D})$ is the pixel intensity at $\mathbf{s}, \mu$ is the mean of the foreground intensity, $\sigma$ is the standard deviation and $f_{\mathbf{s}}$ is some adaptively estimated offset. Details for this formulation can be found in [67]. 


\subsubsection{Motion-Based}

If only moving objects are of interest, matching can be greatly facilitated by adding also this term

$$
E_{m i s_{8}}\left(\mathbf{w} ; \mathcal{H}_{j}, \mathbf{D}\right)=-\sum_{i} \mathcal{M}_{v}\left(\mathbf{v}_{i}(\mathbf{w}) ; \mathbf{D}\right)
$$

where $\mathcal{M}_{v}$ is an indicator function for moving pixels computed based on consecutive image frames $[50,78]$. In general, in an image sequence, there may be several objects moving in different directions. If we intend to track objects moving in a certain direction, a specific indicator function for pixels moving in that direction can be used.

\subsection{User-Controlled Criterion}

Besides model deformation and data mismatch criteria, some user-controlled criteria may also be introduced for better matching. For instance, Cohen et al. [35] pointed out that DM matching may be trapped by some spurious edges. They proposed using user-controlled balloon forces (which act like inflating a balloon), with their directions aligned with the normal vectors of the model contour to prevent matching to spurious edges. The criterion function associated with the balloon force can be formulated as

$$
E_{\text {balloon }}\left(\mathbf{w} ; \mathcal{H}_{j}\right)=-A(\mathbf{w})
$$

where $A(\mathbf{w})$ is the area enclosed by the model contour with parameters $\mathbf{w}$. Other works using the ballooning force include [148] by Xu et al.

As such user-controlled criteria are comparatively heuristic in nature, they will be excluded from our subsequent discussions for coherence. We will only consider model deformation and data mismatch criteria as the major types. 


\section{Regularization}

Model deformation and data mismatch are two often conflicting criteria. The most common way to achieve a trade-off is to define a combined criterion function by a (possibly adaptively) weighted sum of the two. Such a technique, called regularization [133], has long been used for computer vision tasks [115]. Let $\alpha$ denote the regularization parameter. The combined criterion function is

$$
\begin{aligned}
E_{t o t}\left(\mathbf{w}, \alpha ; \mathcal{H}_{j}, \mathbf{D}, \overline{\mathbf{w}}\right) & =\mathcal{Y}\left(E_{\text {def }}\left(\mathbf{w} ; \mathcal{H}_{j}, \overline{\mathbf{w}}\right), E_{m i s}\left(\mathbf{w} ; \mathcal{H}_{j}, \mathbf{D}\right), \alpha\right) \\
& =\alpha E_{\text {def }}\left(\mathbf{w} ; \mathcal{H}_{j}, \overline{\mathbf{w}}\right)+E_{m i s}\left(\mathbf{w} ; \mathcal{H}_{j}, \mathbf{D}\right)
\end{aligned}
$$

From the regularization standpoint, extracting objects from an image, just like many other computer vision tasks, is an ill-posed problem. That is, the problem is underconstrained and hence does not have a unique solution. The term $E_{\text {def }}$ acts as a regularizer to make the extraction (or matching) better-posed. This is especially important when the imagery data is noisy.

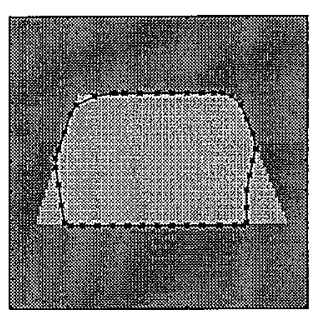

(a) $\alpha=0.02$

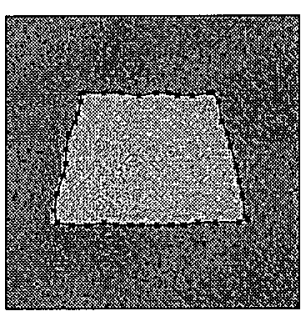

(b) $\alpha=0.02$

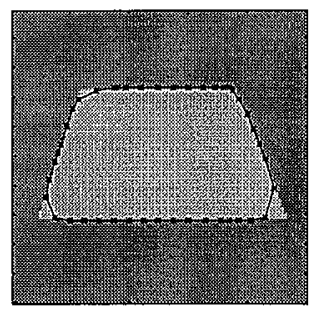

(c) $\alpha=0.001$

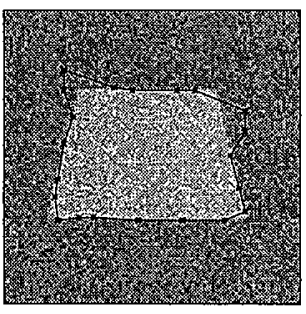

(d) $\alpha=0.001$

Figure 7: Effects of different values of regularization parameter $\alpha$. For the noiseless cases (a) and (c), too large the value of $\alpha$ is less preferable because sharp corners, which in many situations serve as salient features, cannot be detected. For the noisy cases (b) and (d), too small the value of $\alpha$ is less preferable as the model then cannot smooth out the noise and becomes very sensitive to it.

Properly setting the value of $\alpha$ is vital for good matching. Too small the value of $\alpha$ 
implies that the term $E_{m i s}$ dominates and hence the matching result will be very sensitive to noise, while too large the value of $\alpha$ implies that the term $E_{\text {def }}$ dominates, resulting in large discrepancy with the data (Figure 7). Finding an input-independent optimal value of $\alpha$ either through training or by trial and error is one solution. Better matching can also be achieved by systematically decreasing the value of $\alpha$ from large to small, or in other words, allowing the model to be rigid at the beginning to obtain a rough match and then increasingly flexible towards the end for a fine match $[19,52,152,118]$. However, the decreasing rate of $\alpha$ and the corresponding stopping rule still have to be carefully chosen. To achieve optimal regularization by automatically adapting to the input data $\mathbf{D}$, crossvalidation was adopted by Shahraray et al. [126] and Bayesian techniques by Cheung et al. [28]. There also exist some heuristics proposed specifically for specific applications [68].

Besides the standard way of regularization, it is interesting to note that Lai in [82] proposed using a minimax criterion with

$$
E_{\text {tot }}\left(\mathbf{w}, \alpha ; \mathcal{H}_{j}, \mathbf{D}, \overline{\mathbf{w}}\right)=\max \left\{E_{m i s}\left(\mathbf{w} ; \mathcal{H}_{j}, \mathbf{D}\right), E_{\text {def }}\left(\mathbf{w} ; \mathcal{H}_{j}, \overline{\mathbf{w}}\right)\right\}
$$

instead of a weighted combined criterion. It has been shown that under certain convexity assumptions, minimizing such a criterion (which implements the matching process to be discussed in the next section) is equivalent to minimizing the weighted sum criterion with optimal regularization.

\section{$5 \quad$ Matching}

Matching a DM to the data is performed by minimizing the combined criterion function $E_{t o t}$. From a physical point of view, the process can be understood as finding an equilibrium point in the presence of internal and external forces derived from $E_{\text {def }}$ and $E_{m i s}$ (Figure 8). It is often the case that $E_{\text {tot }}$ is a highly nonlinear function and hence contains many spurious local minima. In order for the model parameters to finally converge to the optimal values, 
i.e., $\mathbf{w}^{f}=\mathbf{w}^{*}$ where $\mathbf{w}^{*}=\arg \min _{\mathbf{w}} E_{t o t}\left(\mathbf{w}, \alpha ; \mathcal{H}_{j}, \mathbf{D}, \overline{\mathbf{w}}\right)$, either a local minimizer with good initialization or otherwise a global minimizer is required.

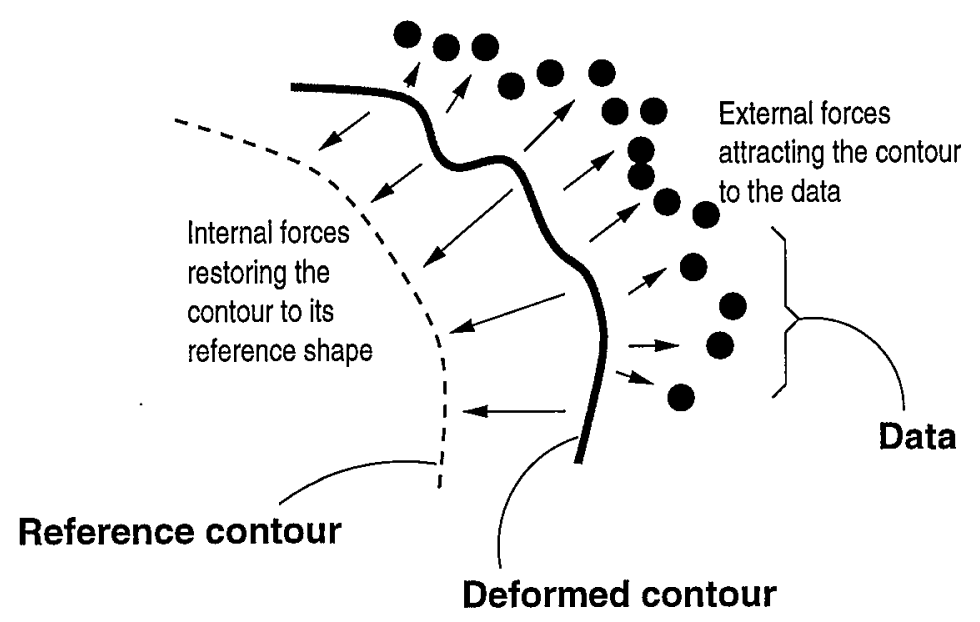

Figure 8: Illustration of DM matching as force balancing in a physical system.

\subsection{Initialization}

To achieve optimal matching such that $\mathbf{w}^{f}=\mathbf{w}^{*}$, good model initialization is known to be important (Figure 9).

If user interaction is allowed, the simplest way is to manually place the initial model close enough to the interested region, e.g., object boundary. To semi-automate the initialization step, Berger et al. [9], for example, proposed to initialize an active contour model by starting with a short snake placed on the desired boundary. The snake then "grows" towards the two ends to extract the whole boundary. With a similar idea, Neuenschwander et al.'s approach [106] starts with a complete snake whose end points are accurately placed at some desired positions. Then, image forces due to the data come into effect progressively, starting from the two end points towards the middle portion of the snake. Such semi-automatic techniques, though useful in some specific applications, fail when the noise level is high or when subjective contours exist. For robust and fully automatic initialization, Lades et al. [81] proposed using a feature-based model attributed by Gabor features to match to the 


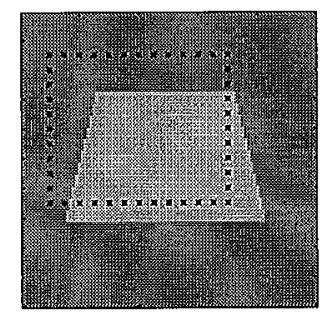

(a) Bad initialization

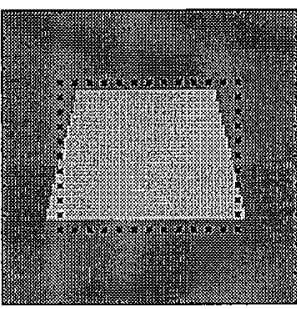

(c) Good initialization

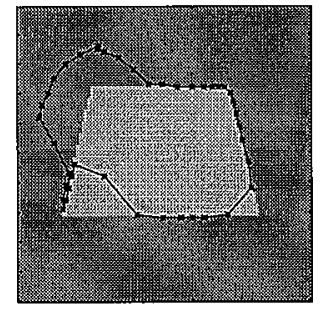

(b) Final match based on bad initialization

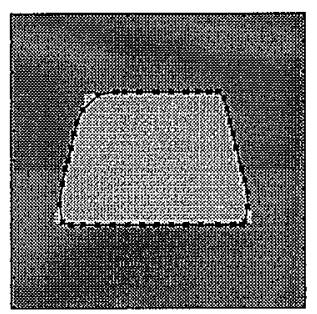

(d) Final match based on good initialization

Figure 9: Effects of different initializations. The object boundary can be successfully extracted only when initialization is good. 
Gabor features extracted from the input image. They succeeded in automatically initializing a human face model in a portrait image. MacCormick et al. [94] demonstrated using random sampling together with a feature-based model to locate objects of interest efficiently, but with the assumption of small degree of scale variation. Amit et al. [3] took advantage of some robust local features to match a graphical hand model to an X-ray image, which is insensitive to initialization. Alternatively, Lai [83] proposed using generalized Hough transform with promising results. Williams et al. [142] and Rowley et al. [121] succeeded in training artificial neural networks to achieve reasonably good initialization.

\subsection{Optimization}

Criterion function optimization is the core engine for model matching. Different optimization methods have been adopted with different degrees of success. As a general comment, global optimization methods provide better results but are time-consuming, while local optimization methods are much more efficient but can only give sub-optimal solutions. Coarse-to-fine multi-resolution optimization is a good trade-off between global and local optimization methods.

\subsubsection{Local Optimization}

\section{a) Optimization in Continuous Domain}

Among different optimization methods, steepest decent $[77,151,127]$ is the simplest one. Based on Kass et al.'s formulation, it is equivalent to solving the Euler equation [77]. To improve the convergence rate, more complex gradient-based methods like conjugate gradient [127], Newton's method and Levenberg-Marquardt method [147] can also be used. Besides, some other methods have also been used, including Powell's direction set method [127], iterated condition mode [67, 78], etc.

b) Optimization in Discrete Grid Domain 
Instead of searching in the $x-y$ continuous space, matching algorithms can also be derived based on the discrete grid of the sampled image. By placing search windows at different locations of the model as illustrated in Figure 10, some discrete local search methods, e.g., greedy search algorithm [143], can be used for criterion function optimization. The advantages of searching in the discrete grid domain include numerical stability and the ease of incorporating hard constraints. For example, a constraint on the minimum distance between two consecutive contour points can easily be introduced by explicitly adding one more condition in the search algorithm. Besides, optimizing a criterion with a very complex non-differential functional form is often a nightmare in the continuous domain, but can still be effectively done by some search methods in the discrete grid domain.

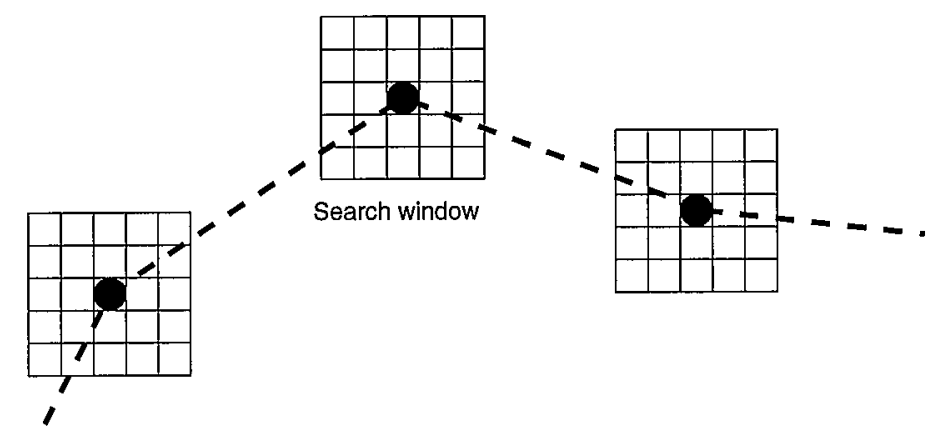

Figure 10: Illustration of optimal boundary extraction in the discrete grid domain with search windows

\subsubsection{Global Optimization}

Local optimization methods can only guarantee sub-optimal solutions. To achieve optimal matching, global optimization methods, like simulated annealing $[73,78,64]$ and genetic algorithms [86], have been used. Alternatively, discrete optimization methods, such as dynamic programming $[2,62]$ and the $A^{*}$ algorithm [37], have also been used. The optimality of these discrete optimization methods depends on the size of the search window and the resolution of the grid (i.e., discretization level). 


\subsubsection{Multi-resolution Optimization}

Global optimization, though can give better results, is known to be computationally expensive. Coarse-to-fine multi-resolution optimization has been found to be more appealing in practice, as on one hand it can escape from many of the local minima and on the other hand the total computational cost required is much less than that of global optimization. Examples of using the coarse-to-fine paradigm can be found in $[145,73,82]$.

\subsubsection{Closed-Form Solutions and Other Heuristics}

It is worth noting that for some special-purpose implementations, closed-form solutions for the optimization problem can be derived [114], where all the sampled input imagery data are taken into account in the criterion formulation step, instead of being explored in an iterative manner during the optimization search process. Besides, there also exist other heuristics proposed for better matching [65].

\subsection{Probabilistic Interpretation}

DM matching, as pointed out by many researchers $[127,50,73]$, can be formulated as MAP estimation under a certain probabilistic interpretation of the process.

From a probabilistic point of view, the deformation of a model $\mathcal{H}_{j}(\mathbf{w})$, quantified by $E_{d e f}\left(\mathbf{w} ; \mathcal{H}_{j}, \overline{\mathbf{w}}\right)$, can be interpreted as the uncertainty that it is indeed deformed from its reference model $\mathcal{H}_{j}(\overline{\mathbf{w}})$. The data mismatch, quantified by $E_{\text {mis }}\left(\mathbf{w} ; \mathcal{H}_{j}, \mathbf{D}\right)$, can be understood as the uncertainty that the input data $\mathbf{D}$ indeed comes from the model $\mathcal{H}_{j}(\mathbf{w})$. Using the Gibbs distribution, such uncertainties can be represented as probabilities, where $E_{\text {def }}$ corresponds to the prior distribution of the model parameters and $E_{m i s}$ corresponds to the likelihood function. Using the Bayes rule, $E_{\text {tot }}$ then corresponds to the posterior distribution. Mathematically, they are written as: 


\section{Prior distribution of $\mathrm{w}$}

$$
p\left(\mathbf{w} \mid \alpha, \mathcal{H}_{j}, \overline{\mathbf{w}}\right)=\frac{1}{Z_{\text {def }}(\alpha)} \exp \left(-\alpha E_{\text {def }}\left(\mathbf{w} ; \mathcal{H}_{j}, \overline{\mathbf{w}}\right)\right.
$$

\section{Likelihood function of $w$}

$$
p\left(\mathbf{D} \mid \mathbf{w}, \mathcal{H}_{j}\right)=\frac{1}{Z_{m i s}(\mathbf{w})} \exp \left(-E_{m i s}\left(\mathbf{w} ; \mathcal{H}_{j}, \mathbf{D}\right)\right)
$$

\section{Posterior distribution of $\mathrm{w}$}

$$
p\left(\mathbf{w} \mid \mathbf{D}, \alpha, \mathcal{H}_{j}, \overline{\mathbf{w}}\right)=\frac{1}{Z_{t o t}(\alpha, \mathbf{D})} \exp \left(-E_{t o t}\left(\mathbf{w} ; \alpha, \mathcal{H}_{j}, \mathbf{D}, \overline{\mathbf{w}}\right)\right.
$$

where $Z_{\text {def }}(\alpha), Z_{m i s}(\mathbf{w})$ and $Z_{t o t}(\alpha, \mathbf{D})$ are the partition functions for normalization and $\alpha$ is the regularization parameter.

Thus, minimizing $E_{t o t}\left(\mathbf{w} ; \alpha, \mathcal{H}_{j}, \mathbf{D}, \overline{\mathbf{w}}\right)$ is equivalent to MAP estimation by maximizing the posterior distribution $p\left(\mathbf{w} \mid \mathbf{D}, \alpha, \mathcal{H}_{j}, \overline{\mathbf{w}}\right)$ with respect to $\mathbf{w}$. As mentioned in Section 1.3, the importance of the probabilistic interpretation is more than just a nice mathematical framework. Many techniques for parameter estimation and hypothesis testing can be borrowed from statistics if such a probabilistic interpretation exists. For example, Revow et al. [118] proposed a matching algorithm based on the EM algorithm, which is originated from statistics for MLE with missing data [47]. Moreover, using a hierarchical Bayesian framework, a formal classification procedure, often referred to as model selection, has also been derived $[82,28]$. This framework will be described in Section 6.1.3.

\section{Classification}

The naive way of performing model-based classification is done by first matching all possible model candidates to the input, then comparing the discriminant measures computed for

\footnotetext{
It should be noted that the EM algorithm is a constrained optimization algorithm.
} 
all the candidates and finally picking the best candidate as the classified output. The classification accuracy and reliability are largely related to the discriminant measure used.

\subsection{Discriminant Measures for Classification}

Different discriminant measures have been adopted for DM-based classification. In general, they can be categorized into the following types: ad hoc distance measures, combined criterion functions, class posterior probabilities and measures based on discriminative classifiers.

\subsubsection{Ad hoc Distance Measures}

Using prior knowledge about specific problems, ad hoc distance measures can sometimes be derived with reasonably good discriminating power. For example, Burr's elastic matching [19] for line drawings used a distance measure based on directional and positional incompatibilities between model and data for classification. Rejection rule was introduced by comparing the values of the discriminant measure for the best and second best candidates. Sclaroff et al. [124] defined a strain energy for classifying the adopted FEM models. The energy value indicates how much a model is deformed after matching (equivalent to a model deformation criterion). The idea of using only a model deformation criterion for classification has also been adopted by Cootes at el. for face recognition [39].

\subsubsection{Combined Criterion Functions}

There is a major difference between an ad hoc distance measure and a combined criterion function. Although both of them can be seen as distance measures, a combined criterion function, besides for classification, also serves as the optimization criterion for matching, which implies the possibility of tight integration of pattern matching and classification into a single step. In fact, it is quite natural and common to use combined criterion functions as discriminant measures $[73,50,81]$, though it is not theoretically justified. 


\subsubsection{Class Posterior Probabilities}

A theoretically sound and disciplined classification method is by comparing the posterior probabilities $\operatorname{Pr}\left(\mathcal{H}_{j} \mid \mathbf{D}\right)$ computed for different classes $[82,69,28]$. If all the model candidates are assumed to be equally probable, i.e., $\operatorname{Pr}\left(\mathcal{H}_{j}\right)=K$ for all $j$ where $K$ is a constant, then maximizing $\operatorname{Pr}\left(\mathcal{H}_{j} \mid \mathbf{D}\right)$ is equivalent to maximizing the likelihood $p\left(\mathbf{D} \mid \mathcal{H}_{j}\right)$. Using the Bayes rule, $p\left(\mathbf{D} \mid \mathcal{H}_{j}\right)$ can be expressed as

$$
p\left(\mathbf{D} \mid \mathcal{H}_{j}\right)=\int \frac{p\left(\mathbf{D} \mid \mathbf{w}, \mathcal{H}_{j}\right) p\left(\mathbf{w} \mid \alpha, \mathcal{H}_{j}\right)}{p\left(\mathbf{w} \mid \mathbf{D}, \alpha, \mathcal{H}_{j}\right)} p\left(\alpha \mid \mathcal{H}_{j}\right) d \alpha
$$

From EQ.(21), it is noted that if an analytical solution can be found for the integration or can be easily computed numerically, there is in fact no need to find the optimal model parameters $\mathbf{w}^{*}$. Instead, the parameter vector is treated as a random vector and is marginalized out. This is equivalent to saying that the matching process is no longer required at all. However, the exact computation of EQ.(21) very often is either too difficult or too computationally demanding. Approximation is generally required. For example, Laplace approximation can be used for approximating the integrand by a multivariate Gaussian function so that only the (sub)optimal values of the parameters $\mathbf{w}$ and $\alpha$ as well as the Hessians about them have to be computed [95]. In that case, the optimal matching and regularization results, $\mathbf{w}^{*}$ and $\alpha^{*}$, are required for the approximation.

\subsubsection{Measures Based on Discriminative Classifiers}

For model-based approaches, it is easy to understand that any inaccuracies in the modeling and criterion function formulation steps can greatly affect the subsequent classification performance. Rather than correcting the inaccuracies which may not be easy, DMs may instead be used only to extract some (possibly noisy) high-level features, like the values of $E_{d e f}$ and $E_{m i s}$ for different models, and the extracted features are then fed into an artificial neural network (ANN) $[125,141]$ or a statistical classifier [66] for subsequent classification. 
The classification performance is optimized by training the classifier so that the effect of the inaccuracies can be reduced. The limitation, again, is the requirement of large quantity of training data for the classifier. Also, whenever there is a new class, the whole set of training data for all classes is often required for re-training the classifier from scratch.

\section{$7 \quad 2 \frac{1}{2} \mathrm{D}$ and $3 \mathrm{D}$ Deformable Models}

Following the development of 2D DMs, many $2 \frac{1}{2} \mathrm{D}$ and $3 \mathrm{D}$ DMs have been proposed in the early 1990s for modeling and recognizing images or 3D objects. The increase in dimension complicates the problem in several aspects. First, modeling an arbitrary $3 \mathrm{D}$ object is much more difficult than modeling an arbitrary $2 \mathrm{D}$ contour. Also, matching a $3 \mathrm{D}$ model to $2 \mathrm{D}$ or 3D data involves a much greater search space, which in turn increases the computational complexity and model initialization difficulty $[45,107]$. In this section, we merely focus on the model representation issue and provide readers pointers to some $2 \frac{1}{2} \mathrm{D}$ and $3 \mathrm{D}$ DMs in the literature.

Research on representing arbitrary 3D objects started in the 1970s, when Binford et al. [12] proposed using generalized cylinders (GC) to represent 3D objects. A GC is a parametric model, which consists of a cross-section curve, a path along which the crosssection curve is swept to form a volume, and a sweeping function which varies the scale of the cross-section along the path. Since then, GCs have been extended for better modeling capabilities. Global shape parameterization using a set of sinusoidal basis functions has also been proposed by Staib at el. [128] as an alternative choice for 3D shape modeling. Another interesting parametric representation of 3D objects is superquadric [131]. By varying a compact set of parameters, an ellipsoid can be continuously deformed to a cube. GCs and superquadrics are both parametric and, subsequently, are limited in representational power. Enhancement can be achieved by superimposing local deformation on them, forming semi- 
parametric models $[131,109]$. In particular, Terzopoulos et al. [131] proposed a deformable superquadric (DS) which matches to the input 3D data by globally deforming a superquadric and at the same time adding local deformation, which is expressed as a linear combination of some basis functions for fitting the details. Based on DSs, many other extensions have been proposed [99, 42, 113]. Besides, Cohen et al. [33] proposed a deformable hyperquadric $(\mathrm{DH})$ for $3 \mathrm{D}$ shape modeling. A hyperquadric is a more general global shape model which includes a superquadric as a special case. Thus a $\mathrm{DH}$ is representationally more powerful than a DS. For other examples of 3D DMs with semi-parametric representation, readers are referred to the works by Pentland et al. [114], Cohen et al. [36] and Donnell et al. [110].

For non-parametric examples, Terzopoulos et al. proposed a symmetry-seeking model for reconstructing a symmetrical $3 \mathrm{D}$ object from a $2 \mathrm{D}$ image [132]. Whitten proposed a $2 \frac{1}{2} \mathrm{D}$ deformable sheet models [139] for matching to $2 \frac{1}{2} \mathrm{D}$ data. Besides, some $3 \mathrm{D}$ non-parametric deformable surface models have also been proposed $[46,34,136]$. While all these works were derived by extending Kass et al.'s active contour models, Feldmar et al. [53] used an approach similar to Wakahara's LAT [138] for parameterizing deformable surfaces. Also, Caunce et al. [22] extended the PDM model (see Section 2.2) to 3D.

Recently, a deformable intensity surface $[104,101,102]$ was proposed for face recognition. This $2 \frac{1}{2} \mathrm{D}$ model is purely non-parametric as the representation is simply a matrix of pixel values. The matching process is analogous to image warping. Simple idea has also been explored by Jones et al. [75].

\section{Applications}

\subsection{Object Detection and Tracking}

DMs have been found to have great potential in many application domains. As mentioned in Section 1.4, DMs are capable of accurately locating non-rigid objects or features in noisy 
images and tracking them through an image sequence $[137,99,153,105,90,41,13,49,78$, $8,62,44,108,103,93,7]$. Such properties are found to be especially useful for medical image analysis, e.g., locating and tracking a shape-varying biological object in a sequence of echocardiographic (ECG), magnetic resonance (MR) or X-ray images $[67,1,80,113,149$, 110, 89, 79, 117] (Figure 11).

Some related applications include precise measurement of some shape-related parameters for medical diagnosis [55] and industrial automatic inspection [140, 70] tasks, image registration $[18,59,60,53]$, model-based coding with very low bit rate [88], etc.

\subsection{Object Correspondence}

Object correspondence (also called signal matching) is a nontrivial but important component in many computer vision tasks, like stereopsis, motion analysis, and constructing 3D models from 2D image slices. The difficulties in obtaining the correspondence between a pair of images lie in the fact that interested objects can be non-rigid, occluded, or very noisy. Using two 2D DMs for the left and right images, which interact either through a true 3D model [6], affine epipolar geometry [25] or a smoothness constraint on the disparity between them [77, 98], promising results on stereo matching have been demonstrated. Other examples of applying DMs to object correspondence were reported by Witkin et al. [145] and Sclaroff et al. [124]. See Figure 12 for an illustration.

\subsection{Object Recognition}

DMs have been applied to the recognition of different non-rigid objects with promising results. The domains include human faces $[16,15,81,96,39,87,104,134]$, gestures $[63,78]$, handwriting $[19,69,66,10,26,138,28,74,30]$, etc. See Figure 13 for a handwriting recognition illustration. 


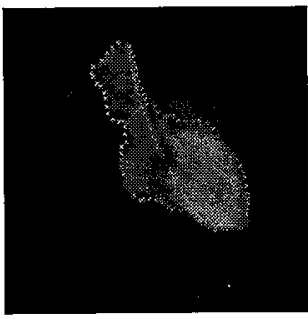

(a)

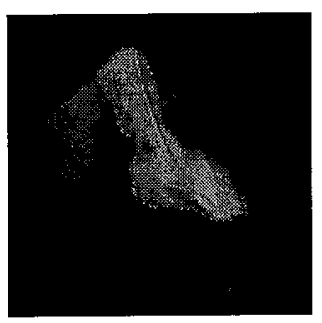

(e)

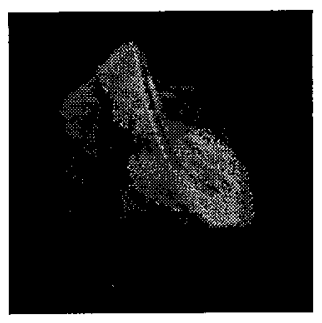

(i)

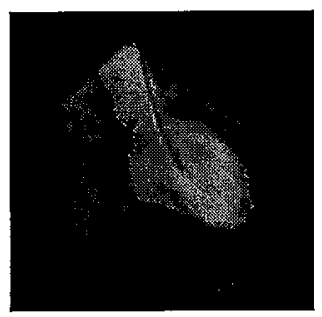

(m)

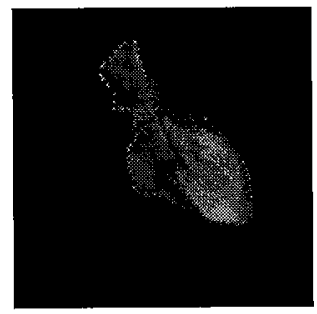

(b)

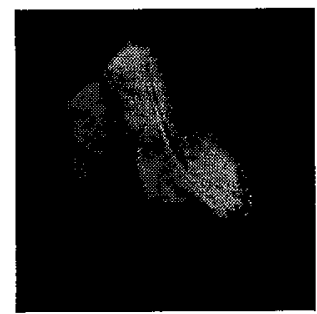

(f)

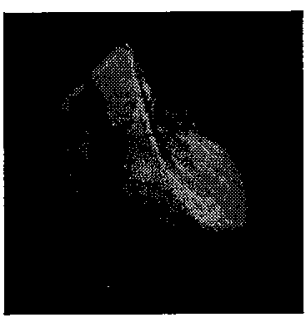

(j)

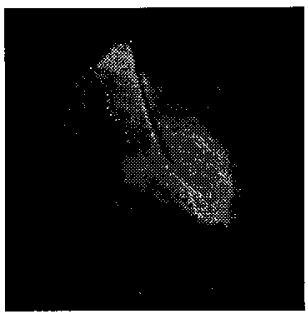

(n)

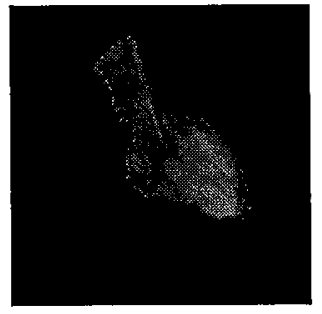

(c)

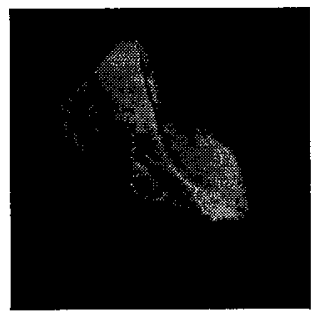

(g)

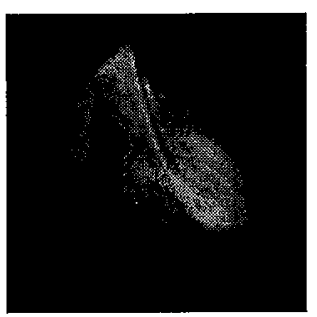

(k)

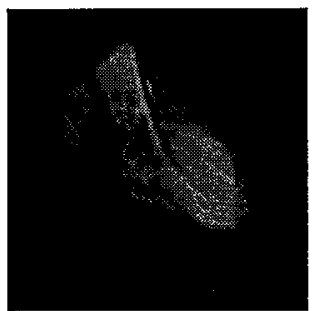

(o)

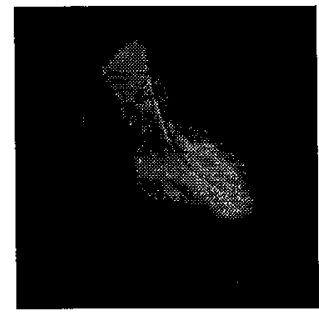

(d)

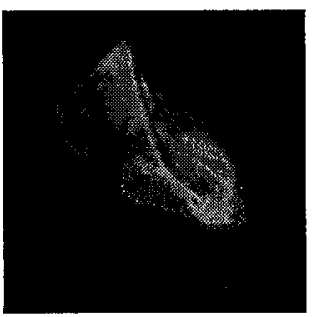

(h)

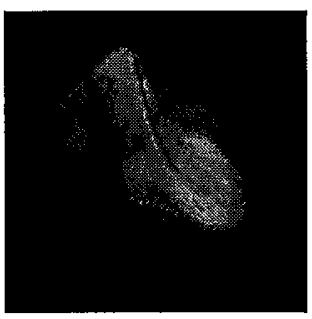

(l)

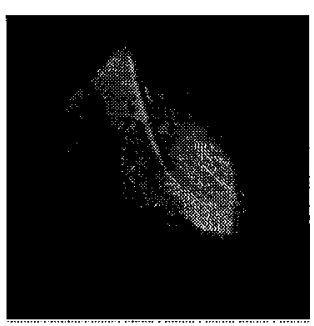

(p)

Figure 11: Tracking a sequence of heart images (using the software package GSNAKE ver. 1.0). The image sequence can be downloaded from ftp://whitechapel.media.mit.edu/pub/images/ and GSNAKE ver. 1.0 from http://www.iti.gov.sg/iti.people/iti_staff/kflai/gsnake.html. 

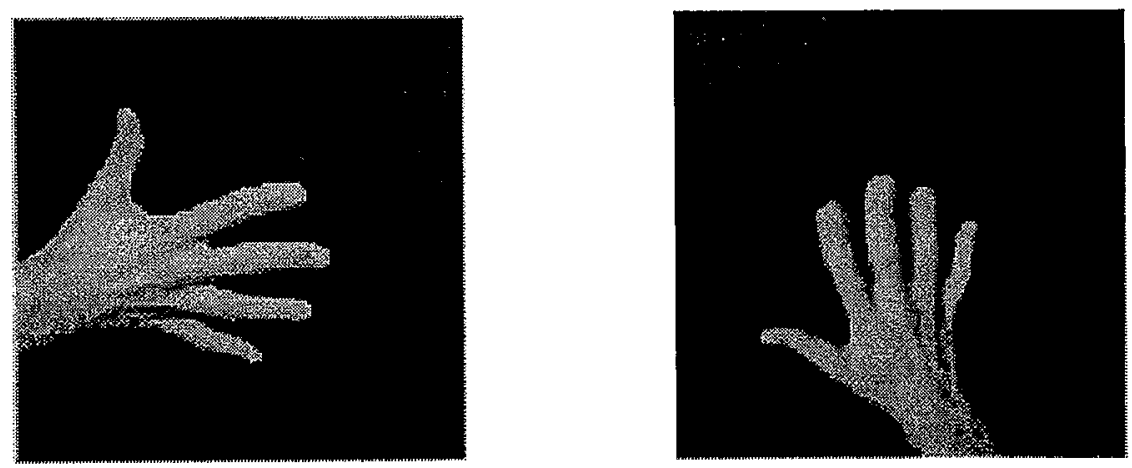

(a) A pair of hand images

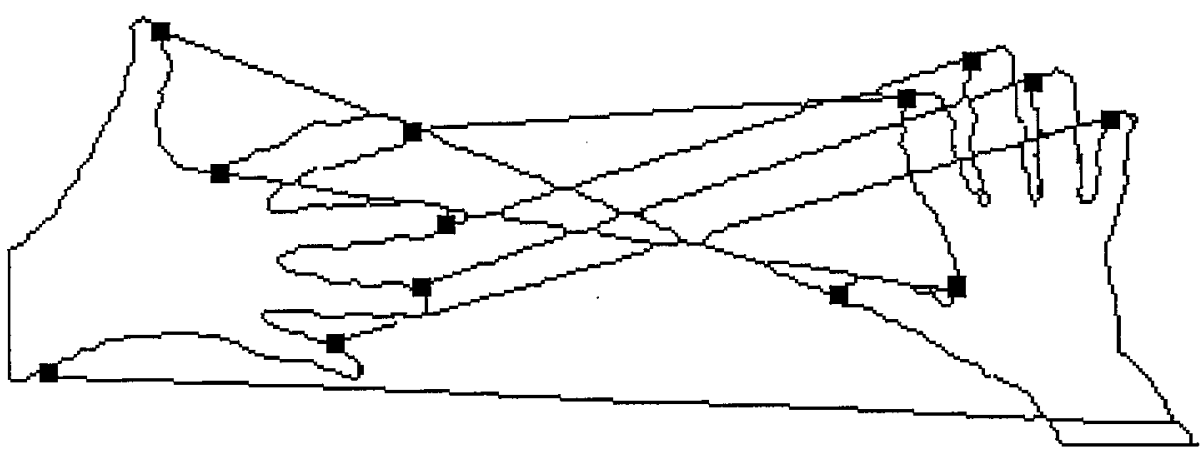

(b) Correspondence between the silhouettes of two hand images

Figure 12: Results of correspondence between two hand images obtained using deformable models [124].

\subsection{Content-based Image Retrieval}

Shape-based image database search is another attractive application domain for DMs. Directly applying DMs for image retrieval is apparently very computationally expensive. Some hybrid approaches are needed to build efficient retrievel systems. Some prototype systems have been constructed. In [155], cues like texture and color extracted from a query are first used to filter out irrelevant images in the database. Then, shape-based deformable template matching is performed between the query shape and the potential images so as to rank them according to shape similarity and output them as the retrieval result. In [11], images in the database are assumed to be preprocessed such that each object of interest has been located by a rectangular bounding box. Besides, a signature file is pre-computed for 


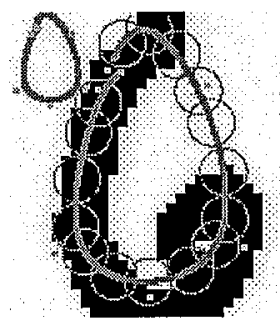

(a) $\mathrm{M} 00 / 0 / 6.39$

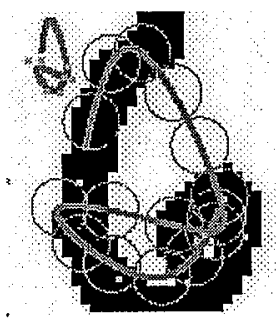

(f) $\mathrm{M} 21 / 2 / 6.50$

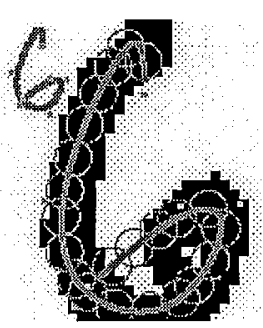

(k) $\mathrm{M} 60 / 6 / 6.16$

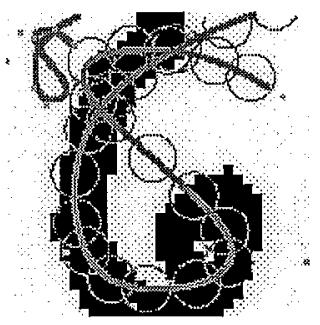

(p) $M 81 / 8 / 6.62$

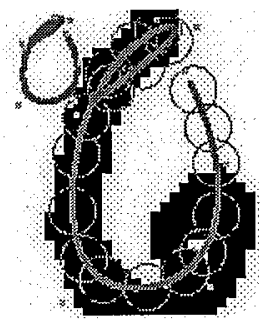

(b) $\mathrm{M} 01 / 0 / 6.40$

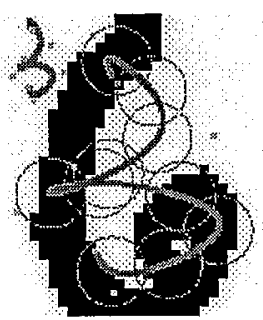

(g) $\mathrm{M} 30 / 3 / 6.76$

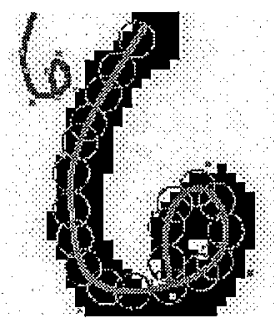

(l) $M 61 / 6 / 6.06^{*}$

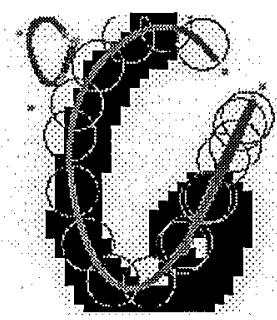

(q) $M 90 / 9 / 6.60$

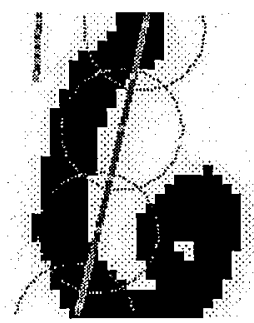

(c) $\mathrm{M} 10 / 1 / 7.03$

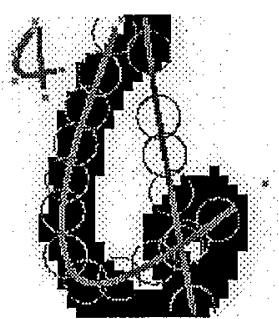

(h) $\mathrm{M} 40 / 4 / 6.30$

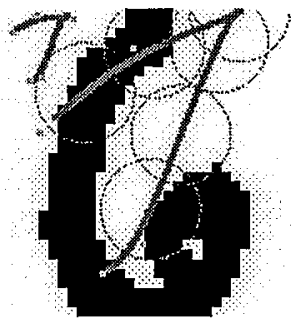

(m) $\mathrm{M} 70 / 7 / 7.19$

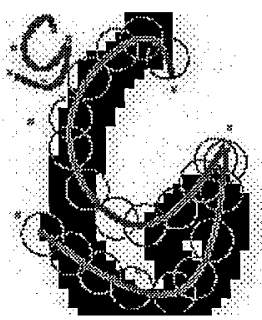

(r) $M 91 / 9 / 6.38$

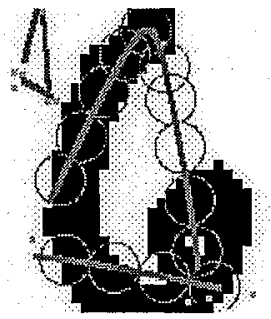

(d) $M 11 / 1 / 6.54$

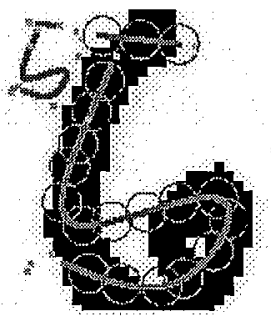

(i) $\mathrm{M} 50 / 5 / 6.19$

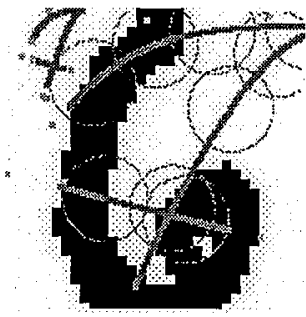

(n) $M 71 / 7 / 7.06$

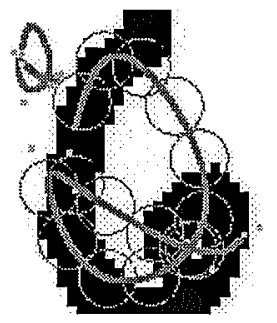

(e) $\mathrm{M} 20 / 2 / 6.49$

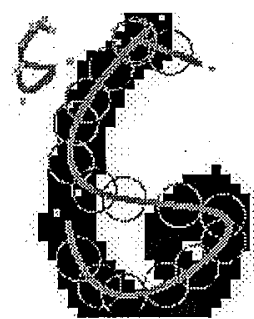

(j) $M 51 / 5 / 6.26$

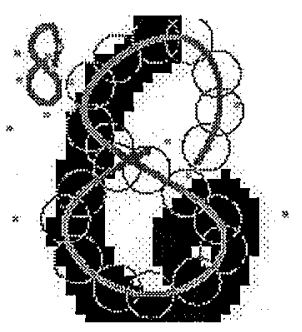

(o) $\mathrm{M} 80 / 8 / 6.48$

Figure 13: Handwritten digit recognition using deformable models. The caption MXX/Y/ZZZZ under each sub-figure is read as: XX-model label, Y-model class, ZZZZmismatch measure. For this example, model with label M61 is found to have the lowest mismatch value and thus the input is classified to be "6". See $[28,29]$ for more details. 
each images, which describes the objects (as bounding boxes) contained and their spatial relationship in a very compact format. Such signature files can allow fast screening to be performed first. The remaining candidate images with similar object spatial relationship are then matched with the query shape for final ranking. In [122], images in the database are assumed to be pre-categorized into, say, $n$ categories, each of which is represented by a deformable prototype. Then, each image is indexed by an $n$-tuple with each element storing a dissimilarity measure (a formulation of "strain" energy) pre-computed by matching the input with a particular deformable prototype. For a query shape, a similar $n$-tuple can be computed and the search can be efficiently done by simply comparing the $n$-tuples.

\section{$9 \quad$ Future Research Directions}

Although DM-based approaches have been found to be promising for non-rigid shape analysis and recognition, there remains much room for further research and development before they can be widely applied to many real-world problems. In this section, some possible future research directions are discussed.

\subsection{Hierarchical Semi-parametric Model Representation}

As mentioned in Section 2, parameteric models typically have lower representational power and non-parameteric models usually have higher computational requirement. A hierarchical semi-parametric model, which adopts a coarse representation (e.g., a wireframe with large and wide grid) for the global shape and a locally smooth representation (e.g., a set of parametric basis functions) for the local deformation, can strike a good balance between representational power and computational efficiency. This direction of designing generalpurpose 3D object representations for both computer vision and computer graphics is worth pursuing. 


\subsection{Topologically Adaptive DMs}

Akin to many parameter estimation algorithms, existing DM matching algorithms mostly assume the use of models with relatively simple topology. This limits their application to the analysis of complex shapes, such as a vessel branching. Also, there does not exist a fixed DM topology which is optimal for all inputs [17]. These motivate the idea of developing topologically adaptive DMs. The adaptability can be achieved through splitting [97, 144] and merging [43] approaches. How to obtain an "optimal" topology for a DM by learning from a given set of training data is still an open question. Another interesting issue is how to establish a probabilistic measure defined for the topological space so that it can be integrated into a probabilistic framework for subsequent inference tasks.

Other than making the model topologically adaptive, Vemuri et al. [135] proposed using pedal curves and surfaces, which are capable of modeling shapes with varying topology. Also, Caselles et al. proposed a geodesic active contour [20], which is represented by the zero level-set of a 3D function. Since there is no restriction on how the zero level-set is formed, the corresponding contour model is topology independent. Such idea has also been extended to the $3 \mathrm{D}$ scenario [21].

\subsection{Guidelines for Criterion Function Formulation}

The choice of criterion functions for model deformation and data mismatch greatly affects the performance of a DM-based system. Although we are still far from reaching a conclusion about whether there exist some universally optimal choices for the criterion functions, the following considerations, summarized from some previous works on DMs, should be worth taking as guidelines for the formulation of criterion functions.

Physical Consideration A model deformation criterion should be formulated to represent well prior knowledge, if available, about the physical properties of the interested 
objects. For example, in a human face model, the mouth component is expected to be more easily deformed than the nose component. Such prior knowledge is important for good matching but is sometimes difficult to incorporate manually when defining the criterion. Training will definitely help. However, collecting training data could be a big problem since it is by far mostly done manually. A fully automatic training scheme, in that scenario, would be very appealing.

Uniqueness and Invariance Consideration Uniqueness and geometric invariance properties are always important for DM-based recognition. In other words, the model representation and the model deformation criterion should be carefully designed to achieve these properties.

Input Feature Consideration The input domain of a data mismatch criterion can be simply image pixels or features extracted by some data-driven algorithms, as described in Section 3.2. The latter choice is generally found to result in more robust matching. The advantage can be understood as the combination of a purely top-down process (DM-based matching) and a purely bottom-up process (feature extraction). However, a bottom-up process may result in the possibility of losing some useful information. So, instead of using just one type of feature, a data mismatch criterion based on different feature types may be used to achieve more robust and reliable results.

Noise Model and Outlier Consideration The data mismatch criterion should be formulated to represent well the noise model of the interested features. A Gaussian noise model is commonly used, but it is not satisfactory in many cases, especially when the input data contains outliers. Robust statistics [72] can be used to modify the data mismatch criterion to discount the effect caused by outliers [154]. In particular, for an application of extracting characters from handwritten cursive script using DMs (Figure 14), it is quite unreasonable to treat the outlier data using a simple Gaussian 
noise model. Using a robust statistical technique called M-estimation, Cheung et al. [27] showed that characters can be correctly extracted even in the presence of outliers.

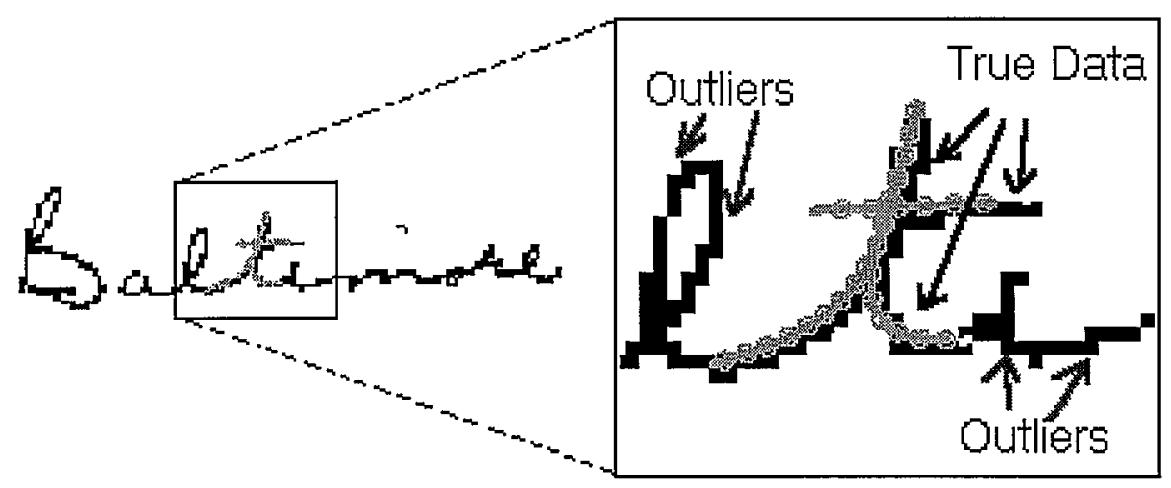

Figure 14: Extraction of the letter ' $t$ ' from closely cluttered characters.

\subsection{More on Matching}

Among all the issues related to optimization, model initialization is still the most difficult yet crucial one. An automatic and efficient initialization method suitable for real-time applications is still lacking in the literature. The situation becomes even worse if the objects to detect are in a complex scene. So far feature-based and neural network-based approaches $[81,118,121]$ show most promising results for some particular applications. With proper initialization, optimization can be performed in a multi-resolution fashion for more efficient matching. Other than software tricks, building a hardware chip for DM matching should be another exciting direction to pursue.

All the discussions so far assume that there is only one DM at a time for matching. In general, the image of a scene can contain several non-rigid objects. Robust statistical approaches, as mentioned in the previous section, try to identify which pixels (data) to regard as outliers for a particular DM. An alternative is to use multiple models for matching, which is more suitable for situations where the number of objects in the scene is known or can be easily computed. This raises an issue of assigning data to multiple DMs. Since 
all the models can deform, which data points to be assigned to which DMs casts a nontrivial assignment problem. It is also non-trival to control the deformation of the different DMs. Some researchers have started working on the assignment problem. Bozma et al. [14] proposed using a game-theoretic approach while Kakadiaris et al. [76] adopted a fuzzy clustering approach.

\subsection{More on Classification}

Compared with matching, DM-based classification is an area that requires much more further research. Although highly accurate recognition has been demonstrated using DM-based methods, one of the major difficulties is again related to computational requirement. Even though a very efficient matching algorithm can be derived, the recognition time is still linear to the size of the model base as long as sequential machines are used, hindering the practical use of this approach for real-time applications. In [29], it was shown that significant speedup without much performance degradation can be achieved if some competition process is introduced at the early stage of matching to eliminate some unlikely model candidates. Such a competition process is believed to be a promising direction for efficient classification. Besides, whether there is a competition process which can achieve both higher accuracy and efficiency is another interesting direction to pursue. In fact, such a competition process in general can also be replaced by other preclassification steps for screening potential candidates for final detailed matching.

Indexing algorithms, such as geometric hashing [85], are known to be efficient for modelbased recognition. The general idea is to gain computational advantage by using additional memory to store precomputed values in the form of hash tables. The development of indexing algorithms has mostly been based on rigid models, where all the object transformations can be solely described by affine transformation. Rigoutsos et al. [119] proposed a modified version which can allow a tiny amount of deformation. For some applications involving 
shapes that can be parameterized by a compact set of parameters, it is possible to extend the indexing algorithms to non-rigid shapes.

Ignoring the implementation concerns, theoretical work on DM-based recognition is still lacking in the literature. Most researchers still perform the classification step using relatively ad hoc methods, instead of following some formal uncertainty reasoning techniques. Combining DMs with a probabilistic framework is so far the most theoretically sound approach. Using such a framework, one can perform classification in a disciplined manner $[82,28]$

\section{Conclusion}

An extensive review of various DM methods for modeling, matching and classifying nonrigid shapes has been presented. Future research directions are also discussed in the paper from the perspectives of representation, criterion function formulation, matching and classification. As of today, the state of the art of DM-based pattern matching is close to full maturity for real-world applications in some confined problem domains, while much more research and experience is still required for the development of DM-based pattern classification.

\section{Acknowledgements}

This research work is supported in part by the Hong Kong Research Grants Council (RGC) under Competitive Earmarked Research Grants HKUST 614/94E and HKUST 746/96E.

\section{References}

[1] A.A. Amini, P. Shi, T. Constable, and K. Johnson, "Energy-minimizing deformable 
grids for tracking tagged MR cardiac images", in Proceedings of Computers in Cardiology, Durham, NC, Oct. 1992, pp. 651-654.

[2] A.A. Amini, T.E. Weymouth, and R.C. Jain, "Using dynamic programming for solving variational problems in vision", IEEE Transactions on Pattern Analysis and Machine Intelligence, vol. 12, no. 9, pp. 855-867, Sept. 1990.

[3] Y. Amit and A. Kong, "Graphical templates for model registration", IEEE Transactions on Pattern Analysis and Machine Intelligence, vol. 18, no. 3, pp. 225-236, Mar. 1997.

[4] K. Arbter, "Affine-invariant Fourier descriptors", in From Pixels to Features, J. C. Simon, Ed., Bonas, France, Aug. 1989, North-Holland.

[5] B. Bascle and R. Deriche, "Features extraction using parametric snakes", in Proceedings of the Eleventh IAPR International Conference on Pattern Recognition, The Hague, Netherlands, Aug. 1992, vol. III, pp. 659-662.

[6] B. Bascle and R. Deriche, "Stereo matching, reconstruction and refinement of 3D curves using deformable contours", in Proceedings of the Fourth International Conference on Computer Vision, Berlin, Germany, Apr. 1993, pp. 421-430.

[7] S. Basu, N. Oliver, and A. Pentland, "3D modeling and tracking of human lip motions", in Proceedings of the Sixth International Conference on Computer Vision, Bombay, India, Jan. 1998, pp. 337-343.

[8] S. Benayoun, N. Ayache, and I. Cohen, "Adaptive meshes and nonrigid motion computation", in Proceedings of the Twelfth IAPR International Conference on Pattern Recognition, Jerusalem, Israel, Oct. 1994, vol. 1, pp. 730-732. 
[9] M. Berger and R. Mohr, "Towards autonomy in active contour models", in Proceedings of the Tenth IAPR International Conference on Pattern Recognition, Atlantic City, NJ, June 1990, pp. 847-851.

[10] J.M. Bertille, "An elastic matching approach applied to digit recognition", in Proceedings of the Second International Conference on Document Analysis and Recognition, Tsukuba Science City, Japan, Oct. 1993, pp. 82-85.

[11] A.D. Bimbo and P. Pala, "Visual image retrieval by elastic matching of user sketches", IEEE Transactions on Pattern Analysis and Machine Intelligence, vol. 19, no. 2, pp. 121-132, Feb. 1997.

[12] T.O. Binford, "Visual perception by computer", in IEEE Conference on Systems and Control, Miami, FL, Dec. 1971.

[13] A. Blake and M. Isard, "3D position, attitude and shape input using video tracking of hands and lips", in Proceedings of the Twenty First International SIGGRAPH Conference, Orlando, Florida, July 1994, pp. 185-192.

[14] H.I. Bozma and J.S. Duncan, "Model-based recognition of multiple deformable objects using a game-theoretic framework", in Proceedings of the Twelfth International Conference on Information Processing in Medical Imaging, Wye, UK, July 1991, pp. $358-372$.

[15] J. Buhmann, M. Lades, and C. Malsburg, "Size and distortion invariant object recognition by hierarchical graph matching", in Proceedings of the International Joint Conference on Neural Networks, Washington, DC, June 1990, vol. 2, pp. 411-416.

[16] J. Buhmann, J. Lange, and C. Malsburg, "Distortion invariant object recognition by matching hierarchically labeled graphs", in Proceedings of the International Joint Conference on Neural Networks, Los Angeles, CA, June 1989, vol. 1, pp. 155-159. 
[17] A.J. Bulpitt and N.D. Efford, "An efficient 3D deformable model with a selfoptimizing mesh", Image and Vision Computing, vol. 14, no. 8, pp. 573-580, Aug. 1996.

[18] D. Burr, "A dynamic model for image registration", Computer Graphics and Image Processing, vol. 15, pp. 102-112, 1981.

[19] D. Burr, "Elastic matching of line drawings", IEEE Transactions on Pattern Analysis and Machine Intelligence, vol. 3, no. 6, pp. 708-713, Nov. 1981.

[20] V. Caselles, R. Kimmel, and G. Sapiro, "Geodesic active contours", International Journal of Computer Vision, vol. 22, no. 2, pp. 61-79, Feb-Mar 1997.

[21] V. Caselles, R. Kimmel, G. Sapiro, and C. Sbert, "Minimal surfaces based object segmentation", IEEE Transactions on Pattern Analysis and Machine Intelligence, vol. 19, no. 4, pp. 394-398, Apr. 1997.

[22] A. Caunce and C.J. Taylor, "3D point distribution models of the cortical sulci", in Proceedings of the Sixth International Conference on Computer Vision, Bombay, India, Jan. 1998, pp. 402-407.

[23] A. Chakraborty, L.H. Staib, and J.S. Duncan, "Deformable boundary finding influenced by region homogeneity", in Proceedings of the IEEE Computer Society Conference on Computer Vision and Pattern Recognition, Seattle, WA, June 1994, pp. $624-627$.

[24] A. Chakraborty, M. Worring, and J.S. Duncan, "On multi-feature integration for deformable boundary finding", in Proceedings of the Fifth International Conference on Computer Vision, Cambridge, MA, June 1995, pp. 846-851. 
[25] T.J. Cham and R. Cipolla, "Stereo coupled active contours", in Proceedings of the IEEE Computer Society Conference on Computer Vision and Pattern Recognition, San Juan, Puerto Rico, June 1997, pp. 1094-1099.

[26] W.T. Chen and T.R. Chou, "A hierarchical deformation model for on-line cursive script recognition", Pattern Recognition, vol. 27, no. 2, pp. 205-219, Feb. 1994.

[27] K.W. Cheung, D.Y. Yeung, and R.T. Chin, "Robust deformable matching for character extraction", Submitted.

[28] K.W. Cheung, D.Y. Yeung, and R.T. Chin, "A unified framework for handwritten character recognition using deformable models", in Proceedings of the Second Asian Conference on Computer Vision, Singapore, Dec. 1995, vol. I, pp. 344-348.

[29] K.W. Cheung, D.Y. Yeung, and R.T. Chin, "Competitive mixture of deformable models for pattern classifification", in Proceedings of the IEEE Computer Society Conference on Computer Vision and Pattern Recognition, San Francisco, CA, June 1996, pp. 613-618.

[30] T.R. Chou and W.T. Chen, "A stochastic representation of cursive Chinese characters for on-line recognition", Pattern Recognition, vol. 30, no. 6, pp. 903-919, 1997.

[31] G.E. Christensen, R.D. Rabbitt, and M.T. Miller, "Deformable templates using large deformation kinematic", IEEE Transactions on Image Processing, vol. 5, no. 10, pp. 1435-1447, Oct. 1996.

[32] W.J. Christmas, J. Kittler, and M. Petrou, "Structural matching in computer vision using probabilistic relaxation", IEEE Transactions on Pattern Analysis and Machine Intelligence, vol. 17, no. 8, pp. 749-764, Aug. 1995. 
[33] I. Cohen and L.D. Cohen, "A hybrid hyperquadric model for 2D and 3D data fitting", Computer Vision and Image Understanding, vol. 63, no. 3, pp. 527-541, May 1996.

[34] I. Cohen, L.D. Cohen, and N. Ayache, "Using deformable surfaces to segment 3D images and infer differential structures", CVGIP: Image Understanding, vol. 56, no. 2, pp. 242-263, Sept. 1992.

[35] L.D. Cohen, "On active contour models and balloons", CVGIP: Image Understanding, vol. 53, no. 2, pp. 211-218, Mar. 1991.

[36] L.D. Cohen and I. Cohen, "Finite element methods for active contour models and balloons for 2D and 3D images", IEEE Transactions on Pattern Analysis and Machine Intelligence, vol. 15, no. 11, pp. 1131-1147, Nov. 1993.

[37] D.B. Cooper, "Maximum likelihood estimation of Markov-process blob boundaries in noisy images", IEEE Transactions on Pattern Analysis and Machine Intelligence, vol. 1 , no. 4, pp. 372-384, Oct. 1979.

[38] T.F. Cootes and C.J. Taylor, "Active shape models - Smart snakes", in Proceedings of the Third British Machine Vision Conference, Leeds, UK, Sept. 1992, pp. 266-275.

[39] T.F. Cootes and C.J. Taylor, "Combining point distribution models with shape models based on finite element analysis", Image and Vision Computing, vol. 13, no. 5, pp. 403-409, July 1995.

[40] T.F. Cootes, C.J. Taylor, A. Lanitis, D.H. Cooper, and J. Graham, "Building and using flexible models incorporating grey-level information", in Proceedings of the Fourth International Conference on Computer Vision, Berlin, Germany, Apr. 1993, pp. $242-246$. 
[41] P.A. Couvignou, N.P. Papanikolopoulos, and P.K. Khosla, "On the use of snakes for 3D robotic visual tracking", in Proceedings of the IEEE Computer Society Conference on Computer Vision and Pattern Recognition, New York, NY, June 1993, pp. 750-751.

[42] D. DeCarlo and D. Metaxas, "Blended deformable models", in Proceedings of the IEEE Computer Society Conference on Computer Vision and Pattern Recognition, Seattle, WA, June 1994, pp. 566-572.

[43] D. DeCarlo and D. Metaxas, "Blended deformable models", IEEE Transactions on Pattern Analysis and Machine Intelligence, vol. 18, no. 4, pp. 443-448, Apr. 1996.

[44] P. Delagnes, J. Benois, and D. Barba, "Active contour approach to object tracking in image sequences with complex background", Pattern Recognition Letters, vol. 16, no. 2, pp. 171-178, Feb. 1995.

[45] H. Delingette, "Initialization of deformable models from 3D data", in Proceedings of the Sixth International Conference on Computer Vision, Bombay, India, Jan. 1998, pp. $311-316$.

[46] H. Delingette, M. Hebert, and K. Ikeuchi, "Deformable surfaces: a free-form shape representation", in Proceedings of SPIE: Geometric Methods in Computer Vision, San Diego, CA, July 1991, vol. 1570, pp. 21-30.

[47] A.P. Dempster, N.M. Laird, and D.B. Rubin, "Maximum-likelihood from incomplete data via the EM algorithm", Journal of the Royal Statistical Society, Series B, vol. 39, pp. 1-38, 1977.

[48] J.Y. Deng and F. Lai, "Region-based template deformation and masking for eyefeature extraction and description", Pattern Recognition, vol. 30, no. 3, pp. 403-419, Mar. 1997. 
[49] S.J. Dickinson and P. Jasiobedzki, "Qualitative tracking of 3D objects using active contour networks", in Proceedings of the IEEE Computer Society Conference on Computer Vision and Pattern Recognition, Seattle, Washington, June 1994, pp. 812817.

[50] M.P. Dubuisson, S. Lakshmanan, and A.K. Jain, "Vehicle segmentation and classification using deformable templates", IEEE Transactions on Pattern Analysis and Machine Intelligence, vol. 18, no. 3, pp. 293-308, Mar. 1996.

[51] R. Durbin, R. Szeliski, and A.L. Yuille, "An analysis of the elastic net approach to the traveling salesman problem", Neural Computation, vol. 1, no. 3, pp. 348-358, 1989.

[52] R. Durbin and D. Willshaw, "An analogue approach to the travelling salesman problem using an elastic net method", Nature, vol. 326, pp. 689-691, 1987.

[53] J. Feldmar and N. Ayache, "Locally affine registration of free-form surfaces", in Proceedings of the IEEE Computer Society Conference on Computer Vision and Pattern Recognition, Seattle, WA, June 1994, pp. 496-501.

[54] M.A. Fischler and R.A. Elschlager, "The representation and matching of pictorial structures", IEEE Transactions on Computers, vol. 22, no. 1, pp. 67-92, Jan. 1973.

[55] Y.L. Fok, J. Chan, and R.T. Chin, "Automated analysis of nerve-cell images using active contour models", IEEE Transactions on Medical Imaging, vol. 15, no. 3, pp. 353-368, June 1996.

[56] J.D. Foley, A.V. Dam, S.K. Feiner, and J.F. Hughes, Computer Graphics, AddisonWesley: Reading, 1991. 
[57] P. Fua and C. Brechbühler, "Imposing hard constraints on deformable models through optimization in orthogonal subspaces", Computer Vision and Image Understanding, vol. 65, no. 2, pp. 148-162, Feb. 1997.

[58] P. Fua and Y.G. Leclerc, "Model driven edge detection", Machine Vision and Applications, vol. 3 , no. 1, pp. 45-56, 1990.

[59] P. Fua and Y.G. Leclerc, "Image registration without explicit point correspondences", in Proceedings of the ARPA Image Understanding Workshop, Monterey, CA, 1994, vol. II, pp. 981-992.

[60] P. Fua and Y.G. Leclerc, "Registration without correspondences", in Proceedings of the IEEE Computer Society Conference on Computer Vision and Pattern Recognition, Seattle, WA, June 1994, pp. 121-127.

[61] K. Fukunaga, Introduction to Statistical Pattern Recognition, Academic Press, San Diego, 2nd edition, 1990.

[62] D. Geiger, A. Gupta, L.A. Costa, and J. Vlontzos, "Dynamic programming for detecting, tracking, and matching deformable contours", IEEE Transactions on Pattern Analysis and Machine Intelligence, vol. 17, no. 3, pp. 294-302, Mar. 1995.

[63] U. Grenander, Y. Chow, and D.M. Keenan, Hands: A Pattern Theoretic Study of Biological Shapes, Springer-Verlag, 1991.

[64] R.P. Grzeszczuk and D.N. Levin, "'Brownian strings": Segmenting images with stochastically deformable contours", IEEE Transactions on Pattern Analysis and Machine Intelligence, vol. 19, no. 10, pp. 1100-1114, Oct. 1997. 
[65] S.R. Gunn and M.S. Nixon, "A robust snake implementation; A dual active contour", IEEE Transactions on Pattern Analysis and Machine Intelligence, vol. 19, no. 1, pp. 63-68, Jan. 1997.

[66] T. Hastie and R. Tibshirani, "Handwritten digit recognition via deformable prototypes", Tech. Rep. Draft, Statistics and Data Analysis Research Department, AT\&T Bell Laboratories, Murray Hill, NJ, USA, July 1992.

[67] I.L. Herlin, "A deformable region model using stochastic processes applied to echocardiographic image", in Proceedings of the IEEE Computer Society Conference on Computer Vision and Pattern Recognition, Champaign, IL, June 1992, pp. 534-539.

[68] I.L. Herlin, C. Nguyen, and C. Graffigne, "Stochastic segmentation of ultrasound images", in Proceedings of the Eleventh IAPR International Conference on Pattern Recognition, The Hague, Netherlands, Aug. 1992, vol. 1, pp. 289-292.

[69] G.E. Hinton, C.K.I. Williams, and M.D. Revow, "Adaptive elastic models for handprinted character recognition", in Advances in Neural Information Processing Systems, J.E. Moody, S.J. Hanson, and R.P. Lippmann, Eds., vol. 4, pp. 512-519. Morgan Kaufmann, 1992.

[70] S. Hirai, H. Wakamatsu, and K. Iwota, "Modeling of deformable thin parts for their manipulation", in Proceedings of the International Conference on Robotics and Automation, San Diego, CA, May 1994, pp. 2955-2960.

[71] Z. Huang and F.S. Cohen, "Affine-invariant B-spline moments for curve matching", in Proceedings of the IEEE Computer Society Conference on Computer Vision and Pattern Recognition, Seattle, WA, June 1994, pp. 490-495.

[72] P.J. Huber, Robust Statistics, Wiley: New York, 1981. 
[73] A.K. Jain, Y. Zhong, and S. Lakshmanan, "Object matching using deformable templates", IEEE Transactions on Pattern Analysis and Machine Intelligence, vol. 18, no. 3, pp. 267-278, Mar. 1996.

[74] A.K. Jain and D. Zongker, "Representation and recognition of handwritten digits using deformable templates", IEEE Transactions on Pattern Analysis and Machine Intelligence, vol. 19, no. 12, pp. 1386--1390, Dec. 1997.

[75] M.J. Jones and T. Poggio, "Multidimensional morphable models", in Proceedings of the Sixth International Conference on Computer Vision, Bombay, India, Jan. 1998, pp. 683-688.

[76] I. Kakadiaris, D. Metaxas, and R. Bajcsy, "Inferring 2D object structure from the deformation of apparent contours", Computer Vision and Image Understanding, vol. 65, no. 2, pp. 129-147, Feb. 1996.

[77] M. Kass, A. Witkin, and D. Terzopoulos, "Snakes: Active contour models", International Journal of Computer Vision, vol. 1, no. 4, pp. 321-331, 1987.

[78] C. Kervrann and F. Heitz, "A hierarchical statistical framework for the segmentation of deformable objects in image sequences", in Proceedings of the IEEE Computer Society Conference on Computer Vision and Pattern Recognition, Seattle, WA, June 1994, pp. $724-728$.

[79] Y. Kita, "Elastic-model driven analysis of several views of a deformable cylindrical object", IEEE Transactions on Pattern Analysis and Machine Intelligence, vol. 18, no. 12, pp. 1150-1162, Dec. 1996.

[80] S. Kumar and D. Goldgof, "Automatic tracking of SPAMM grid and the estimation of deformation parameters from cardiac MR images", IEEE Transactions on Medical Imaging, vol. 13, no. 1, pp. 122-132, Mar. 1994. 
[81] M. Lades, J.C. Vorbruggen, J. Buhmann, J. Lange, C.V.D. Malsburg, R.P. Wurtz, and W. Konen, "Distortion invariant object recognition in the dynamic link architecture", IEEE Transactions on Computers, vol. 42, no. 3, pp. 300-311, Mar. 1993.

[82] K.F. Lai, Deformable contours: Modeling, extraction, detection and classification, $\mathrm{PhD}$ thesis, Electrical and Computer Engineering Department, University of Wisconsin-Madison, 1994.

[83] K.F. Lai and R.T. Chin, "On regularization, formulation and initialization of the active contour models (snakes)", in Proceedings of the First Asian Conference on Computer Vision, 1993, pp. 542-545.

[84] K.F. Lai and R.T. Chin, "Deformable contours: Modeling and extraction", IEEE Transactions on Pattern Analysis and Machine Intelligence, vol. 17, no. 11, pp. 10841090, Nov. 1995.

[85] Y. Lamdan and H.J. Wolfson, "Geometric hashing: A general and efficient modelbased recognition scheme", in Proceedings of the Second International Conference on Computer Vision, Tampa, FL, Dec. 1988, pp. 238-249.

[86] A. Lanitis, "Locating facial features using genetic algorithms", in Proceedings of the International Conference on Digital Signal Processing, Limassol, Cyprus, 1995, pp. $520-525$.

[87] A. Lanitis, C.J. Taylor, and T.F. Cootes, "Automatic face identification system using flexible appearance models", Image and Vision Computing, vol. 13, no. 5, pp. 393-401, June 1995.

[88] A. Lanitis, C.J. Taylor, and T.F. Cootes, "Automatic interpretation and coding of face images using flexible models", IEEE Transactions on Pattern Analysis and Machine Intelligence, vol. 19, no. 7, pp. 743-756, July 1997. 
[89] G.F. Lea and A. Gupta, "The use of hybrid models to recover cardiac wall motion in tagged MR images", in Proceedings of the IEEE Computer Society Conference on Computer Vision and Pattern Recognition, San Francisco, CA, June 1996, pp. 625-630.

[90] F. Leymarie and M.D. Levin, "Tracking deformable objects in the plane using an active contour model", IEEE Transactions on Pattern Analysis and Machine Intelligence, vol. 15, no. 6, pp. 617-634, June 1993.

[91] F. Leymarie and M.D. Levine, "Simulating the grassfire transform using an active contour model", IEEE Transactions on Pattern Analysis and Machine Intelligence, vol. 14, no. 1, pp. 56-75, Jan. 1992.

[92] C.Y. Liou and H.C. Yang, "Handprinted character recognition based on spatial topology distance measurement", IEEE Transactions on Pattern Analysis and Machine Intelligence, vol. 18, no. 9, pp. 941-945, Sept. 1996.

[93] J. Luettin and N. A. Thacker, "Speechreading using probabilistic models", Computer Vision and Image Understanding, vol. 65, no. 2, pp. 163-178, Feb. 1997.

[94] J. MacCormick and Andrew Blake, "A probabilistic contour discriminant for object localization", in Proceedings of the Sixth International Conference on Computer Vision, Bombay, India, Jan. 1998, pp. 390-395.

[95] D.J.C. MacKay, "Bayesian interpolation", Neural Computation, vol. 4, no. 3, pp. 415-447, 1992.

[96] K. Matsuno, S. Tsuji, and C.W. Lee, "Recognition of human facial expressions using 2D physical model", in Proceedings of the IEEE Workshop on Visualization and Machine Vision, Seattle, WA, June 1994, pp. 74-82. 
[97] T. McInerney and D. Terzopoulos, "Topologically adaptable snakes", in Proceedings of the Fifth International Conference on Computer Vision, Cambridge, MA, June 1995, pp. 840-845.

[98] S. Menet, P. Saint-Marc, and G. Medioni, "B-snakes: Implementation and application to stereo", in Artificial Intelligence and Computer Vision. Proceedings of the Seventh Israeli Conference, Ramat Gan, Israel, Dec. 1990.

[99] D. Metaxas and D. Terzopoulos, "Constrained deformable superquadrics and nonrigid motion tracking", in Proceedings of the IEEE Computer Society Conference on Computer Vision and Pattern Recognition, Maui, HI, June 1991, pp. 337-343.

[100] M.I. Miller, G.E. Christensen, Y. Amit, and U. Grenander, "Mathematical textbook of deformable neuroanatomies", Proceedings of the National Academy of Sciences USA, vol. 90, no. 24, pp. 11944-11948, Dec. 1993.

[101] B. Moghaddam, C. Nastar, and A. Pentland, "Bayesian face recognition using deformable intensity surfaces", in Proceedings of the IEEE Computer Society Conference on Computer Vision and Pattern Recognition, San Francisco, CA, June 1996, pp. 638-645.

[102] B. Moghaddam, W. Wahid, and A. Pentland, "Beyond eigenfaces: Probabilistic matching for face recognition", to appear in Proceedings of the Third IEEE International Conference on Automatic Face and Gesture Recognition, Apr. 1998.

[103] C. Nastar and N. Ayache, "Frequency-based non-rigid motion analysis: Application to four dimensional medical images", IEEE Transactions on Pattern Analysis and Machine Intelligence, vol. 18, no. 11, pp. 1067-1079, Nov. 1996. 
[104] C. Nastar and A. Pentland, "Matching and recognition using deformable intensity surfaces", in Proceedings of the IEEE International Symposium on Computer Vision, Coral Gables, FL, Nov. 1995, pp. 223-228.

[105] C. Naster and N. Ayache, "Fast segmentation, tracking and analysis of deformable objects", in Proceedings of the Fourth International Conference on Computer Vision, Berlin, Germany, June 1993, pp. 275-279.

[106] W. Neuenschwander, P. Fua, G. Székely, and O. Kübler, "Initializing snakes", in Proceedings of the IEEE Computer Society Conference on Computer Vision and Pattern Recognition, Seattle, WA, June 1994, pp. 658-663.

[107] W. Neuenschwander, P. Fua, G. Székely, and O. Kübler, "Velcro surfaces: Fast initialization of deformable models", Computer Vision and Image Understanding, vol. 65 , no. 2, pp. 237-245, Feb. 1997.

[108] C.W. Ngo, S. Chan, and K.F. Lai, "Motion tracking and analysis of deformable objects by generalized active contour models", in Proceedings of the Second Asian Conference on Computer Vision, Singapore, Dec. 1995, vol. 3, pp. 442-446.

[109] T. O'Donnel, T.E. Boult, S.F. Xi, and A. Gupta, “The extruded generalized cylinder: A deformable model for object recovery", in Proceedings of the IEEE Computer Society Conference on Computer Vision and Pattern Recognition, Seattle, WA, June 1994, pp. 174-181.

[110] T. O'Donnell, T. Boult, and A. Gupta, "Global models with parametric offsets as applied to cardiac motion recovery", in Proceedings of the IEEE Computer Society Conference on Computer Vision and Pattern Recognition, San Francisco, CA, June 1996, pp. 293-299. 
[111] B. Olstad and A.H. Torp, "Encoding of a priori information in active contour models", IEEE Transactions on Pattern Analysis and Machine Intelligence, vol. 18, no. 9, pp. 863-872, Sept. 1996 .

[112] P. Padeva, J. Serrat, and E. Martí, "A snake for model-based segmentation", in Proceedings of the Fifth International Conference on Computer Vision, Cambridge, MA, June 1995, pp. 816-821.

[113] J. Park, D. Metaxas, and A. Young, "Deformable models with parameter functions: Application to heart-wall modeling", in Proceedings of the IEEE Computer Society Conference on Computer Vision and Pattern Recognition, Seattle, WA, June 1994, pp. $437-442$.

[114] A. Pentland and S. Sclaroff, "Closed-form solutions for physically based shape modeling and recognition", IEEE Transactions on Pattern Analysis and Machine Intelligence, vol. 13, no. 7, pp. 715-729, July 1991.

[115] T. Poggio, V. Torre, and C. Koch, "Computational vision and regularization theory", Nature, vol. 317, pp. 314-319, Sept. 1985.

[116] A.R. Pope, "Model-based object recognition: A survey of recent research", Tech. Rep. 94-04, Computer Science Department, University of British Columbia, Jan. 1994.

[117] P. Radeva, A.A. Amini, and J. Huang, "Deformable B-solids and implicit snakes for 3D localization and tracking of SPAMM MRI data", Computer Vision and Image Understanding, vol. 66, no. 2, pp. 163-178, May 1997.

[118] M. Revow, C.K.I. Williams, and G.E. Hinton, "Using generative models for handwritten digit recognition", IEEE Transactions on Pattern Analysis and Machine Intelligence, vol. 18, no. 6, pp. 592-606, June 1996. 
[119] I. Rigoutsos and R. Hummel, "A Bayesian approach to model matching with geometric hashing", Computer Vision and Image Understanding, vol. 62, no. 1, pp. 11-26, July 1996.

[120] R. Ronfard, "Region-based strategies for active contour models", International Journal of Computer Vision, vol. 13, no. 2, pp. 229-251, 1994.

[121] H.A. Rowley, S. Baluja, and T. Kanade, "Neural network-based face detection", in Proceedings of the IEEE Computer Society Conference on Computer Vision and Pattern Recognition, San Francisco, CA, June 1996, pp. 203-208.

[122] S. Sclaroff, "Deformable prototypes for encoding shape categories in image database", Pattern Recognition, vol. 30, no. 4, pp. 627-641, Apr. 1997.

[123] S. Sclaroff and A. Pentland, "Object recognition and categorization using modal matching", in Proceedings of the Second CAD-Based Vision Workshop, Champion, PA, Feb. 1994, pp. 258-265.

[124] S. Sclaroff and A. Pentland, "Modal matching for correpondence and recognition", IEEE Transactions on Pattern Analysis and Machine Intelligence, vol. 17, no. 6, pp. 545-561, June 1995.

[125] A.W. Senior, Off-line cursive handwriting recognition using recurrent neural networks, PhD thesis, University of Cambridge, Sept. 1994.

[126] B. Shahraray and D.J. Anderson, "Optimal estimation of contour properties by crossvalidated regularization", IEEE Transactions on Pattern Analysis and Machine Intelligence, vol. 11 , no. 6 , pp. 600-610, June 1989. 
[127] L.H. Staib and J.S. Duncan, "Boundary finding with parametrically deformable models", IEEE Transactions on Pattern Analysis and Machine Intelligence, vol. 14, no. 11, pp. 1061-1075, Nov. 1992.

[128] L.H. Staib and J.S. Duncan, "Model-based deformable surface finding for medical images", IEEE Transactions on Medical Imaging, vol. 15, no. 5, pp. 720-731, Oct. 1996.

[129] J. Subrahmonia, D. Keren, and D.B. Cooper, "Recognizing mice, vegetables and hand printed characters based on implicit polynomials, invariants and Bayesian methods", in Proceedings of the Fourth International Conference on Computer Vision, Berlin, Germany, June 1993, pp. 320-324.

[130] D. Terzopoulos, "Deformable models", Visual Computer, vol. 4, no. 6, pp. 306-331, 1988.

[131] D. Terzopoulos and D. Metaxas, "Dynamic 3D models with local and global deformations: Deformable superquadrics", IEEE Transactions on Pattern Analysis and Machine Intelligence, vol. 13, no. 7, pp. 703-714, July 1991.

[132] D. Terzopoulos, A. Witkin, and M. Kass, "Symmetry-seeking models and 3D object reconstruction", International Journal of Computer Vision, vol. 1, no. 3, pp. 211-221, Oct. 1987.

[133] A.N. Tikhonov, "Regularization of incorrectly posed problems", Soviet Mathematic DOKL, vol. 4, pp. 1624-1627, 1963.

[134] R.G. Uhl and N.D.V. Lobo, "A framework for recognizing a facial image from a police sketch", in Proceedings of the IEEE Computer Society Conference on Computer Vision and Pattern Recognition, San Francisco, CA, June 1996, pp. 586-593. 
[135] B.C. Vemuri and Y. Guo, "Snake pedals: Geometric models with physics-based control", in Proceedings of the Sixth International Conference on Computer Vision, Bombay, India, Jan. 1998, pp. 427-432.

[136] B.C. Vemuri and R. Malladi, "Intrinsic parameters for surface representation using deformable models", IEEE Transactions on System, Man and Cybernetics, vol. 23, no. 2, pp. 614-623, Mar./Apr. 1993.

[137] J.B. Waite and W.J. Welsh, "Head boundary location using snakes", British Telecommunication Technology Journal, vol. 8, no. 3, pp. 127-136, July 1990.

[138] T. Wakahara, "Shape matching using LAT and its application to handwritten numeral recognition", IEEE Transactions on Pattern Analysis and Machine Intelligence, vol. 16, no. 6, pp. 618-629, June 1994.

[139] G. Whitten, "Scale space tracking and deformable sheet models for computational vision", IEEE Transactions on Pattern Analysis and Machine Intelligence, vol. 15, no. 7 , pp. 697-706, July 1993.

[140] B. Widrow, "The "rubber-mask" technique - I. Pattern measurement and analysis", Pattern Recognition, vol. 5, pp. 175-197, 1973.

[141] C.K.I. Williams, Combining deformable models and neural networks for handprinted digit recognition, $\mathrm{PhD}$ thesis, Department of Computer Science, University of Toronto, Ontario, Canada, 1994.

[142] C.K.I. Williams, M. Revow, and G.E. Hinton, "Instantiating deformable models with a neural net", Computer Vision and Image Understanding, vol. 68, no. 1, pp. 120-126, Oct. 1997. 
[143] D.J. Williams and M. Shah, "A fast algorithm for active contours and curvature estimation", CVGIP: Image Understanding, vol. 55, no. 1, pp. 14-26, Jan. 1992.

[144] J. Williams and L. Wolff, "Analysis of the pulmonary vascular tree using differential geometry based vector fields", Computer Vision and Image Understanding, vol. 65, no. 2, pp. 226-236, Feb. 1997.

[145] A. Witkin, D. Teropoulos, and M. Kass, "Signal matching through scale space", International Journal of Computer Vision, vol. 1, no. 4, pp. 133-144, 1987.

[146] M. Worring, W.M. Smeulders, L.H. Staib, and J.S. Duncans, "Parameterized feasible boundaries in gradient vector fields", Computer Vision and Image Understanding, vol. 63, no. 1, pp. 135-144, Jan. 1996.

[147] X. Xie, R. Subhakar, and H. Zhuang, "On improving eye feature extraction using deformable templates", Pattern Recognition, vol. 27, no. 6, pp. 791-799, 1994.

[148] G. Xu, E. Segawa, and S. Tsuji, "Robust active contours with insensitive parameters", Pattern Recognition, vol. 27, no. 7, pp. 879-884, 1994.

[149] A. Young, C. Chang, and L. Axel, "Semi-automatic tracking of myocardial motion in MR tagged images", IEEE Transactions on Medical Imaging, vol. 14, no. 3, pp. 422-433, Sept. 1995.

[150] A.L. Yuille, "Generalized deformable models, statistical physics, and matching problems", Neural Computation, vol. 2, no. 1, pp. 1-24, 1990.

[151] A.L. Yuille, D.S. Cohen, and P.W. Hallinan, "Feature extraction from faces using deformable templates", in Proceedings of the IEEE Computer Society Conference on Computer Vision and Pattern Recognition, San Diego, CA, June 1989, pp. 104-109. 
[152] A.L. Yuille, D.S. Cohen, and P. Hallinan, "Feature extraction from faces using deformable templates", International Journal of Computer Vision, vol. 8, no. 2, pp. 99-111, Aug. 1992.

[153] A.L. Yuille, K. Honda, and C. Peterson, "Particle tracking by deformable templates", in Proceedings of the International Joint Conference on Neural Networks, Seattle, WA, July 1991, pp. 7-12.

[154] A. Yuille, C. Peterson, and K. Honda, "Deformable templates, robust statistics and Hough transforms", in Proceedings of the SPIE: Geometric Methods in Computer Vision, 1991, vol. 1570, pp. 166-173.

[155] Y. Zhong and A.K. Jain, "Object localization using color, texture and shape", in Proceedings of the Workshop on Energy Minimization Methods in Computer Vision and Pattern Recognition, Venice, May 1997, pp. 279-294.

[156] S.C. Zhu and A. Yuille, "Region competition: Unifying snakes, region growing, and Bayes/MDL for multiband image segmentation", IEEE Transactions on Pattern Analysis and Machine Intelligence, vol. 18, no. 9, pp. 884-900, Sept. 1996. 